\title{
Ultra-processed food consumption in adults across Europe
}

\author{
Elly Mertens ${ }^{1} \odot \cdot$ Chiara Colizzi $^{1} \cdot$ José L. Peñalvo ${ }^{1}$
}

Received: 7 September 2021 / Accepted: 29 October 2021 / Published online: 3 December 2021

(c) The Author(s) 2021

\begin{abstract}
Purpose The purpose of this study is todescribe ultra-processed food and drinks (UPFDs) consumption, and associations with intake of total sugar and dietary fibre, and high BMI in adults across Europe.

Methods Using food consumption data collected by food records or 24-h dietary recalls available from the European Food Safety Authority (EFSA) Comprehensive European Food Consumption Database, the foods consumed were classified by the level of processing using the NOVA classification. Diet quality was assessed by data linkage to the Dutch food composition tables (NEVO) and years lived with disability for high BMI from the Global Burden of Disease Study 2019. Bivariate groupings were carried out to explore associations of UPFDs consumption with population intake of sugar and dietary fibre, and BMI burden, visualised by scatterplots.

Results The energy share from UPFDs varied markedly across the 22 European countries included, ranging from 14 to $44 \%$, being the lowest in Italy and Romania, while the highest in the UK and Sweden. An overall modest decrease (2-15\%) in UPFDs consumption is observed over time, except for Finland, Spain and the UK reporting increases (3-9\%). Fine bakery wares and soft drinks were most frequently ranked as the main contributor. Countries with a higher sugar intake reported also a higher energy share from UPFDs, as most clearly observed for UPF $(r=0.57, p$ value $=0.032$ for men; and $r=0.53$, $p$ value $=0.061$ for women). No associations with fibre intake or high BMI were observed.

Conclusion Population-level UPFDs consumption substantially varied across Europe, although main contributors are similar. UPFDs consumption was not observed to be associated with country-level burden of high BMI, despite being related to a higher total sugar intake.
\end{abstract}

Keywords Ultra-processed foods $\cdot$ Food consumption survey $\cdot$ Europe $\cdot$ NOVA classification $\cdot$ Diet quality

\section{Introduction}

Food processing, in particular the degree and purpose of the processing, is recognised to be an important determinant of food's nutrient profile, and, therefore, diet quality and population's health [1]. In public health nutrition, the NOVA classification system is regarded as an internationally recognised method for grouping foods according to the nature, extent, and purpose of the industrial processing [2]. The NOVA system classifies all foods and food products in four groups: (1) unprocessed or minimally processed foods, (2) processed culinary ingredients, (3) processed foods, and (4) ultra-processed foods and drinks (UPFDs) [3, 4]. As the

Elly Mertens

ellymertens@itg.be

1 Unit of Non-Communicable Diseases, Department of Public Health, Institute of Tropical Medicine, Antwerp, Belgium
Food Agriculture Organisation (FAO) states: 'UPFDs are the formulation of ingredients, mostly of exclusive industrial use, typically created by series of industrial techniques processes' [2]. Such ingredients and processes designed for the manufacture of UPFDs intend to extend their shelf life but also make them profitable, palatable, attractive and easy-toconsume $[1,2,5]$. However, these foods often have a suboptimal nutritional profile, are energy dense, i.e. they are low in fibre and micronutrients, but high in saturated fats, salt, and sugars inducing a high glycaemic load, hence best to avoid or minimise their consumption $[1,2,5]$. Through this suboptimal profile, diets rich in UPFDs may therefore increase the risk of elevated body mass index (BMI) and contribute to the burden of non-communicable diseases (NCDs) [6]. Emerging evidence from observational cross-sectional and cohort studies has identified positive associations between UPFDs consumption and at least one adverse NCD outcome $[7,8]$, such as high BMI [7], type 2 diabetes [9] as well as 
a higher risk of cardiovascular disease [10] and all-cause mortality [11].

Previous research using total food/drink volume sales per capita from Euromonitor including 80 countries showed that UPFD volume sales is the highest in Western Europe, North America, and Australasia, while also alarmingly increasing in other world regions [12]. Baked goods, including cakes, pastries, industrial breads, and soft drinks ranked among the top contributors to sales of UPFDs [12]. In Europe, UPFDs contributed to an average of $25 \%$ of the total dietary energy with the lowest estimates observed in Portugal and Italy and the highest in Germany and the UK, as estimated from the household budget survey data from 19 European countries [13]. Only a few studies from Belgium [14], France [15], Portugal [16], and the UK [17, 18] have used national representative individual-level dietary data reporting a total proportion of daily energy intake from UPFDs of 30, 31, 24 , and $55 \%$, respectively, with the highest share for baked goods and confectionary, processed meats, and soft drinks [13-15].

To date, a comprehensive overview of how much UPFDs contribute to the diets across all of Europe, as estimated from national individual-level dietary survey data, is lacking. This study aims to characterize European food consumption patterns in terms of their consumption of UPFDs, including the identification of the most consumed UPFDs and changes over time where possible, using aggregated national dietary survey data as available in the European Food Safety Authority (EFSA) Comprehensive European Food Consumption Database. In addition, the present study aims to analyse whether high consumption of UPFDs is associated with poorer dietary quality, as operationalised by the dietary intakes of fibre and sugar and the population burden of BMI.

\section{Materials and methods}

\section{Food consumption data}

Country-level publicly available dietary data estimated from individual-level dietary surveys were obtained from the Comprehensive European Food Consumption Database developed and maintained by the EFSA since 2011 [19]. Countries with national dietary survey data collected for at least 2 days by means of food records or 24-h dietary recalls were selected for the study. From the 25 European countries reporting survey dietary data to EFSA, the 22 countries for which adult food consumption data were available for at least 2 days were selected, excluding Bulgaria, Poland, and Slovakia [19]. The most recent survey year for each country was used to retrieve food consumption by sex, based on the individual mean consumption of the total survey period, although this year varied considerably between countries, i.e. from 2003 for Czech Republic and Hungary to 2017 for Slovenia. Food consumption data were collected by means of at least two 24-h recalls in 15 countries (i.e. Austria, Belgium, Croatia, Cyprus, Czech Republic, Estonia, Finland, France, Germany, Greece, Latvia, the Netherlands, Portugal, Slovenia, Spain), of which six of them were supplemented by either a food frequency questionnaire (i.e. Belgium and France), food propensity questionnaire (i.e. Latvia, Portugal and Slovenia) or lifestyle questionnaire covering supplement use and alcohol consumption (i.e. the Netherlands), and seven countries solely used food records as dietary assessment method (i.e. Denmark, Hungary, Ireland, Italy, Romania, Sweden, and the UK) with the number of days varying between 3 and 7 days.

Summary statistics of food consumption data reported in grams/day and presented according the sixth level of the 'Exposure Hierarchy' of the food classification and description system FoodEx2 [20] were retrieved for the adult population (aged 18-64 years). A number of 2287 different food items coded with a unique FoodEx2-code were consumed in the 22 European countries, and countries reporting on average 565 different food items (with a range from 289 to 995).

\section{Classification of foods according to the NOVA classification}

The foods consumed across Europe and retrieved from EFSA, were classified as 'unprocessed or minimally processed', 'processed culinary ingredients', 'processed', and 'ultra-processed' according to the NOVA classification [3, $5,21]$ (Supplementary Table 1). This classification relied on the coding details of FoodEx2, and on the level of food disaggregation when reporting food consumption. For example, composite dishes, fine bakery wares, doughs and pre-mixes, dairy-based desserts like puddings (whether home-prepared or not), that are included as such in FoodEx2, were not disaggregated into core ingredients, and were all classified as ultra-processed. Alcoholic beverages were kept as a separate group, as they were initially not included in the NOVA classification [2].

\section{Linkage with food composition databases}

The collected food consumption data from EFSA, as coded by FoodEx2, was linked with detailed information on nutritional composition, using the Dutch Food Composition Database (NEVO) [22]. This linkage was initiated by the Dutch Food Consumption Survey 2012-2016 [23], where the foods consumed were coded by NEVO and FoodEx2. Foods reported by the European countries included in the present study were matched to the NEVO code that most closely resembled the sixth level of description of the 


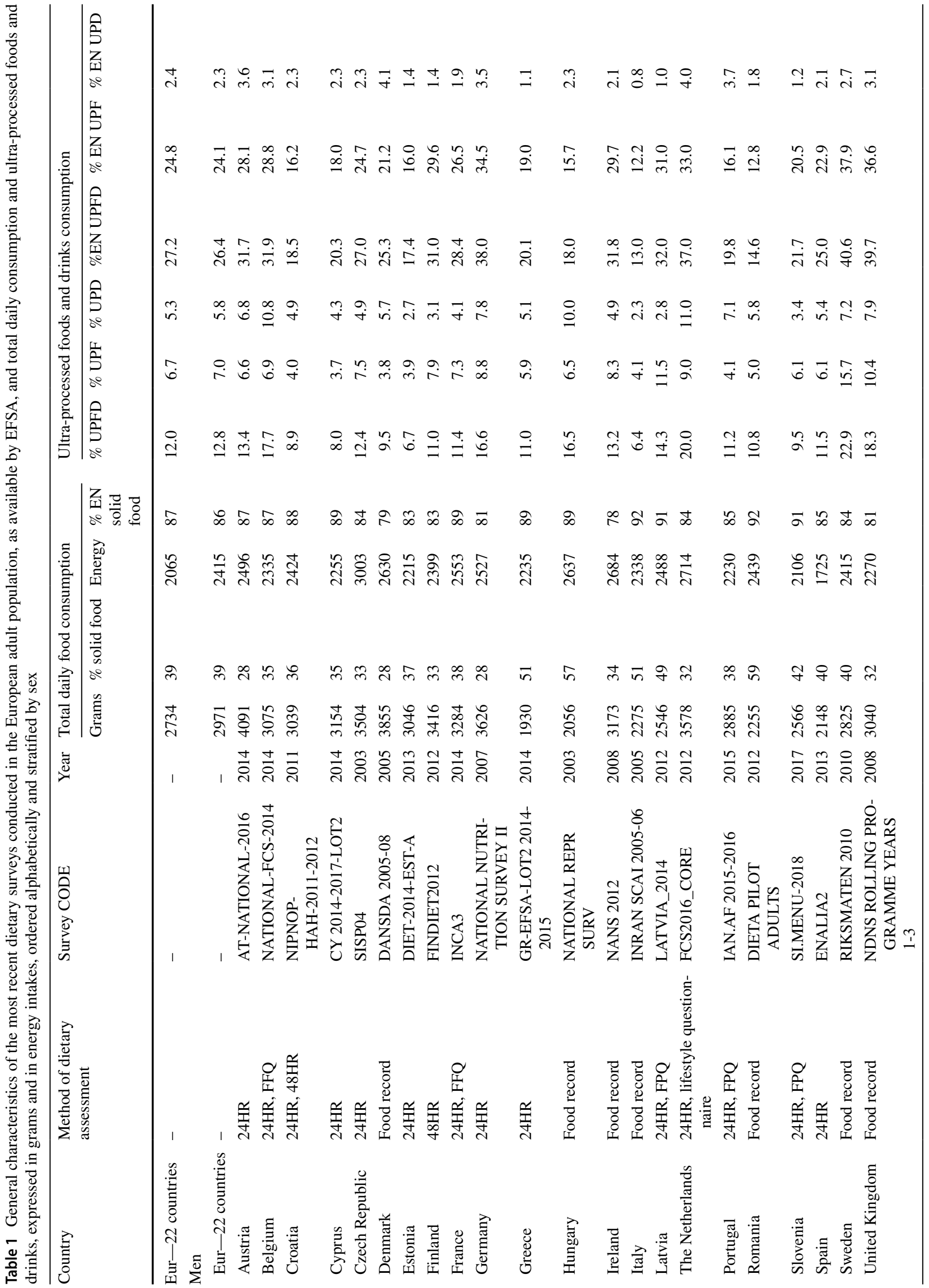




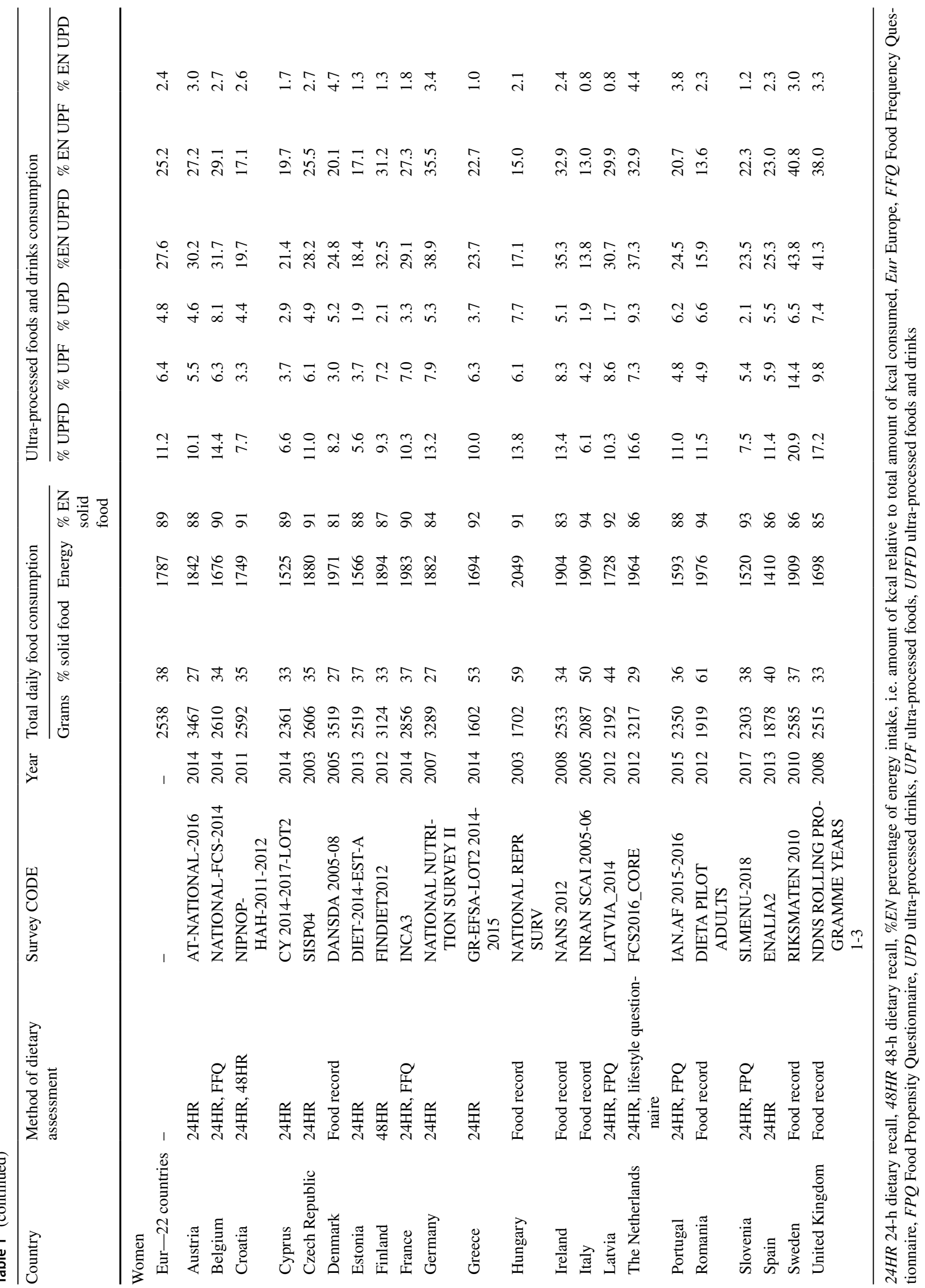


FoodEx2 'Exposure Hierarchy'. This linkage with NEVO allowed the calculation of the dietary composition, and in particular the energy content of the diet for the calculation of the proportion of energy derived from UPFDs, as well as the fibre and sugar content of the diet, as two simple measures of carbohydrate intake and diet quality, also directly associated with body weight and BMI [24, 25].

\section{Countries burden of high BMI}

Countries' burden of high BMI was obtained from the latest release (2019) of the Global Burden of Disease (GBD) Study [26]. The GBD global health data exchange (GBDx) tool was used to extract age-standardised rates of Years Lived with Disability (YLD) (per 100,000) for high BMI, defined as BMI $\geq 25 \mathrm{~kg} / \mathrm{m}^{2}$, for men and women separately and in the year matching the dietary survey, for each country [27]. This disability-adjusted measure, capturing incidence as well as duration of the disability and its associated disability weight, reflects the non-fatal burden of high BMI, hereby providing insight to the severity of living with that condition at the country level.

\section{Analysis}

Using the sixth level of the FoodEx2 'Exposure Hierarchy', the consumption of UPFDs was calculated, expressed as the proportion of daily food intake from UPFDs and the proportion of daily energy intake from UPFDs. The consumption of UPFDs was further segregated according to their physical form (solid vs liquid) into foods (UPFs) and drinks (UPDs), because of their possible implications for food choices, energy balance and body weight management [28]. UPFs included crackers and similar additional bread products, cereal bars, flakes and popped cereals, fine bakery wares, doughs and pre-mixes, nut spreads, fruit chips and chocolate coated dried fruit, meat specialities (meat spread/pate), sausages, fish specialities (fish paste), sweetened or flavoured dairy products, cheese spreads, manufactured eggs, sweetening ingredients (e.g. polyols) and table-top sweeteners, chocolate, candies and confectionary, sweet bars, spoonable water-/dairy-based desserts, margarines and other blended fats and oils, solid foods for particular diets and food supplements, meat imitates, composite dishes (including readyto-eat meals and salads), seasoning, sauces and condiments, and other ingredients. UPDs included flavoured or sweetened milk and yoghurt drinks, milkshakes, fruit and vegetable juices (not 100\% from named source), soft drinks, diet soft drinks, hot cocoa beverages, oral rehydration and carbohydrate-electrolyte solutions, milk imitates, and soups.

Subsequently, the mean contribution of the above-mentioned food subgroups to the dietary share of UPFDs by sex was calculated to identify the foods with the highest contribution to the consumption of UPFDs, with the top five of UPFs and the top three of UPDs identified for the UPFDs expressed in grams per day and in energy per day. For the 11 countries with recurrent dietary surveys over time, i.e. Austria, Belgium, Denmark, Finland, France, Latvia, Ireland, the Netherlands, Spain, Sweden, and the United Kingdom, we analysed their changes in UPFDs consumption, provided that the same dietary assessment method was used for dietary data collection, i.e. either the 24-h recall or food record for the recurrent dietary surveys.

Bivariate groupings were established by considering overlapping of the tertiles for measures of the dietary energy share of UPFDs, and measures of diet quality, represented by dietary fibre and total sugar intake, and of population burden of high BMI. Potential relationships were visualised in scatterplots, using colours to depict the different methods of dietary assessment, and were described using Spearman' rank correlation coefficient $(r)$ with $p$ values adjusted for multiple testing according to Sidak, stratified by the physical form of the food (e.g. solid vs liquid) and sex. A two-sided $p$ value below 0.05 was considered as statistically significant, and all analyses were conducted in STATA (Release SE 16.1/SE. College Station, TX: StataCorp LP).

\section{Results}

\section{Consumption of ultra-processed foods and drinks}

Table 1 shows for each country the total food consumption in grams per day and in energy per day, and the UPFD consumption as expressed in proportion of daily food intake and of energy intake, stratified by sex. The average consumption of UPFDs in adults across Europe was $328 \mathrm{~g} /$ day and the average proportion of daily food consumption amount from UPFDs was $12.0 \%$. The average energy intake from UPFDs was $562 \mathrm{kcal} /$ day, representing an average share of total energy intake of $27.2 \%$ (Table 1 ).

The proportion of daily foodconsumption amount deriving from UPFDs for men ranged from to $6.4 \%$ (Italy) and $6.7 \%$ (Estonia) to $20.0 \%$ (the Netherlands) and $22.9 \%$ (Sweden), of which on average around 55\% was coming from UPFs. The consumption amount of UPDs was, however, higher than that of UPFs in Belgium, Denmark, Hungary, the Netherlands, Portugal and Romania (2-4\% higher) and similar in Austria, Croatia, Cyprus, Estonia, Germany, Greece, Italy, Romania, and Spain. The share of dietary energy coming from UPFDs for men ranged from 12.9\% (Italy) and $14.6 \%$ (Romania) to $39.7 \%$ (the UK) and 40.6\% (Sweden), with on average around $90 \%$ of the dietary energy from UPFDs was coming from UPFs.

Similarly, for women, proportion of daily food consumption amount from UPFDs was low for Estonia (5.7\%) and Italy $(6.1 \%)$, and high for the Netherlands $(16.6 \%)$, the 
Table 2 The top five ultra-processed foods consumed by European adults, as available by EFSA, ordered alphabetically and stratified by sex, expressed as the percentage of daily energy from ultra-processed foods

\begin{tabular}{|c|c|c|c|c|c|}
\hline & \multicolumn{5}{|l|}{ Top five } \\
\hline & 1 & 2 & 3 & 4 & 5 \\
\hline Eur-22 countries & $\begin{array}{l}\text { Fine bakery wares } \\
(26.9 \%)\end{array}$ & Sausages (12.5\%) & $\begin{array}{l}\text { Composite dishes } \\
(9.5 \%)\end{array}$ & Margarines (7.9\%) & Sauces $(7.6 \%)$ \\
\hline \multicolumn{6}{|l|}{ Men } \\
\hline Eur-22 countries & $\begin{array}{l}\text { Fine bakery wares } \\
\quad(25.2 \%)\end{array}$ & Sausages (15.5\%) & $\begin{array}{l}\text { Composite dishes } \\
(9.7 \%)\end{array}$ & Margarines $(8.1 \%)$ & Sauces $(7.9 \%)$ \\
\hline Austria & $\begin{array}{l}\text { Fine bakery wares } \\
\quad(37 \%)\end{array}$ & Sausages (11\%) & Sauces $(10 \%)$ & Chocolate (8\%) & Composite dishes (7\%) \\
\hline Belgium & $\begin{array}{l}\text { Fine bakery wares } \\
(24 \%)\end{array}$ & Sauces $(15 \%)$ & Chocolate (10\%) & Margarines (10\%) & Sausages $(9 \%)$ \\
\hline Croatia & Sausages (41\%) & $\begin{array}{l}\text { Fine bakery wares } \\
(13 \%)\end{array}$ & Chocolate (7\%) & $\begin{array}{l}\text { Crackers and addi- } \\
\text { tional bread products } \\
(6 \%)\end{array}$ & Margarines (5\%) \\
\hline Cyprus & $\begin{array}{l}\text { Fine bakery wares } \\
\quad(37 \%)\end{array}$ & $\begin{array}{l}\text { Crackers and addi- } \\
\text { tional bread products } \\
(18 \%)\end{array}$ & Breakfast cereals $(7 \%)$ & $\begin{array}{l}\text { Composite dishes } \\
(5 \%)\end{array}$ & Sauces $(4 \%)$ \\
\hline Czech Republic & $\begin{array}{l}\text { Fine bakery wares } \\
(30 \%)\end{array}$ & Sausages $(28 \%)$ & $\begin{array}{l}\text { Composite dishes } \\
\text { (12\%) }\end{array}$ & Margarines (4\%) & $\begin{array}{l}\text { Crackers and additional } \\
\text { bread products }(4 \%)\end{array}$ \\
\hline Denmark & Margarines (30\%) & Sausages (10\%) & Chocolate (9\%) & $\begin{array}{l}\text { Fine bakery wares } \\
(7 \%)\end{array}$ & Sauces $(6 \%)$ \\
\hline Estonia & Sausages (28\%) & $\begin{array}{l}\text { Fine bakery wares } \\
(17 \%)\end{array}$ & Margarines (9\%) & Chocolate $(8 \%)$ & Sauces $(8 \%)$ \\
\hline Finland & Margarines (27\%) & $\begin{array}{l}\text { Fine bakery wares } \\
(18 \%)\end{array}$ & Sauces $(10 \%)$ & Sausages $(10 \%)$ & $\begin{array}{l}\text { Sweetened/flavoured } \\
\text { dairy products (9\%) }\end{array}$ \\
\hline France & $\begin{array}{l}\text { Fine bakery wares } \\
(38 \%)\end{array}$ & Sauces $(13 \%)$ & Sausages $(9 \%)$ & $\begin{array}{l}\text { Crackers and addi- } \\
\text { tional bread products } \\
(8 \%)\end{array}$ & $\begin{array}{l}\text { Sweetened/flavoured } \\
\text { dairy products }(8 \%)\end{array}$ \\
\hline Germany & $\begin{array}{l}\text { Fine bakery wares } \\
(23 \%)\end{array}$ & Sausages (15\%) & $\begin{array}{l}\text { Composite dishes } \\
(13 \%)\end{array}$ & Sauces $(11 \%)$ & Margarines (9\%) \\
\hline Greece & $\begin{array}{l}\text { Fine bakery wares } \\
\quad(39 \%)\end{array}$ & $\begin{array}{l}\text { Crackers and addi- } \\
\text { tional bread products } \\
(20 \%)\end{array}$ & Sauces (11\%) & Margarines (5\%) & Breakfast cereals $(4 \%)$ \\
\hline Hungary & Sausages (39\%) & Margarines (16\%) & $\begin{array}{l}\text { Fine bakery wares } \\
(9 \%)\end{array}$ & Chocolate (6\%) & $\begin{array}{c}\text { Crackers and additional } \\
\text { bread products }(5 \%)\end{array}$ \\
\hline Ireland & $\begin{array}{l}\text { Fine bakery wares } \\
(22 \%)\end{array}$ & Margarines (14\%) & $\begin{array}{l}\text { Composite dishes } \\
(12 \%)\end{array}$ & Sauces $(12 \%)$ & Breakfast cereals $(10 \%)$ \\
\hline Italy & $\begin{array}{l}\text { Fine bakery wares } \\
(44 \%)\end{array}$ & Sausages (17\%) & $\begin{array}{l}\text { Composite dishes } \\
(8 \%)\end{array}$ & $\begin{array}{l}\text { Water-/dairy-based } \\
\text { desserts }(7 \%)\end{array}$ & $\begin{array}{l}\text { Crackers and additional } \\
\text { bread products }(5 \%)\end{array}$ \\
\hline Latvia & $\begin{array}{l}\text { Fine bakery wares } \\
(31 \%)\end{array}$ & Sausages (17\%) & $\begin{array}{l}\text { Composite dishes } \\
(17 \%)\end{array}$ & Sauces $(16 \%)$ & Chocolate (6\%) \\
\hline The Netherlands & $\begin{array}{l}\text { Fine bakery wares } \\
(18 \%)\end{array}$ & $\begin{array}{l}\text { Composite dishes } \\
(17 \%)\end{array}$ & Sauces $(12 \%)$ & Margarines (12\%) & Chocolate (7\%) \\
\hline Portugal & $\begin{array}{l}\text { Fine bakery wares } \\
(37 \%)\end{array}$ & Sausages $(8 \%)$ & $\begin{array}{l}\text { Composite dishes } \\
(8 \%)\end{array}$ & Breakfast cereals $(6 \%)$ & $\begin{array}{l}\text { Water-/dairy-based des- } \\
\text { serts }(5 \%)\end{array}$ \\
\hline Romania & Sausages (27\%) & $\begin{array}{l}\text { Composite dishes } \\
(17 \%)\end{array}$ & $\begin{array}{l}\text { Fine bakery wares } \\
(11 \%)\end{array}$ & $\begin{array}{l}\text { Crackers and addi- } \\
\text { tional bread products } \\
(10 \%)\end{array}$ & Margarines (7\%) \\
\hline Slovenia & $\begin{array}{l}\text { Fine bakery wares } \\
(36 \%)\end{array}$ & Sausages (23\%) & $\begin{array}{l}\text { Crackers and addi- } \\
\text { tional bread products } \\
(7 \%)\end{array}$ & Sauces $(6 \%)$ & Chocolate (6\%) \\
\hline Spain & $\begin{array}{l}\text { Fine bakery wares } \\
(36 \%)\end{array}$ & $\begin{array}{l}\text { Crackers and addi- } \\
\text { tional bread products } \\
(15 \%)\end{array}$ & Sausages (12\%) & $\begin{array}{l}\text { Sweetened/flavoured } \\
\text { dairy products }(7 \%)\end{array}$ & Sauces $(6 \%)$ \\
\hline
\end{tabular}


Table 2 (continued)

\begin{tabular}{|c|c|c|c|c|c|}
\hline & \multicolumn{5}{|l|}{ Top five } \\
\hline & 1 & 2 & 3 & 4 & 5 \\
\hline Sweden & $\begin{array}{l}\text { Composite dishes } \\
\text { (42\%) }\end{array}$ & $\begin{array}{l}\text { Fine bakery wares } \\
\quad(14 \%)\end{array}$ & Sauces $(10 \%)$ & Margarines (7\%) & Sausages $(6 \%)$ \\
\hline United Kingdom & $\begin{array}{l}\text { Composite dishes } \\
\text { (32\%) }\end{array}$ & $\begin{array}{l}\text { Fine bakery wares } \\
\quad(15 \%)\end{array}$ & Sauces $(11 \%)$ & Margarines (8\%) & Sausages $(8 \%)$ \\
\hline \multicolumn{6}{|l|}{ Women } \\
\hline Eur-22 countries & $\begin{array}{l}\text { Fine bakery wares } \\
\quad(28.2 \%)\end{array}$ & Sausages $(9.5 \%)$ & $\begin{array}{l}\text { Composite dishes } \\
(9.1 \%)\end{array}$ & Margarines $(9.1 \%)$ & Sauces $(7.2 \%)$ \\
\hline Austria & $\begin{array}{l}\text { Fine bakery wares } \\
(42 \%)\end{array}$ & Sauces $(11 \%)$ & Chocolate (10\%) & $\begin{array}{l}\text { Composite dishes } \\
(6 \%)\end{array}$ & Sausages $(6 \%)$ \\
\hline Belgium & $\begin{array}{l}\text { Fine bakery wares } \\
(29 \%)\end{array}$ & Sauces (13\%) & Chocolate (10\%) & Margarines (9\%) & Sausages $(7 \%)$ \\
\hline Croatia & Sausages $(23 \%)$ & $\begin{array}{l}\text { Fine bakery wares } \\
\quad(16 \%)\end{array}$ & Chocolate (9\%) & Margarines (9\%) & $\begin{array}{l}\text { Crackers and additional } \\
\text { bread products }(8 \%)\end{array}$ \\
\hline Cyprus & $\begin{array}{l}\text { Fine bakery wares } \\
(40 \%)\end{array}$ & $\begin{array}{l}\text { Breakfast cereals } \\
(11 \%)\end{array}$ & $\begin{array}{l}\text { Crackers and addi- } \\
\text { tional bread products } \\
(11 \%)\end{array}$ & $\begin{array}{l}\text { Composite dishes } \\
(6 \%)\end{array}$ & Chocolate (5\%) \\
\hline Czech Republic & $\begin{array}{l}\text { Fine bakery wares } \\
(36 \%)\end{array}$ & Sausages (13\%) & $\begin{array}{l}\text { Composite dishes } \\
(12 \%)\end{array}$ & Margarines (6\%) & $\begin{array}{c}\text { Crackers and additional } \\
\text { bread products }(5 \%)\end{array}$ \\
\hline Denmark & Margarines (28\%) & Chocolate (10\%) & $\begin{array}{l}\text { Fine bakery wares } \\
(7 \%)\end{array}$ & $\begin{array}{l}\text { Candies, confection- } \\
\text { ary }(6 \%)\end{array}$ & Sausages $(6 \%)$ \\
\hline Estonia & $\begin{array}{l}\text { Fine bakery wares } \\
(23 \%)\end{array}$ & Sausages (17\%) & Chocolate (11\%) & Margarines (8\%) & Sauces $(7 \%)$ \\
\hline Finland & Margarines (26\%) & $\begin{array}{l}\text { Fine bakery wares } \\
(20 \%)\end{array}$ & $\begin{array}{l}\text { Sweetened/flavoured } \\
\text { dairy products }(11 \%)\end{array}$ & Sauces (7\%) & Chocolate (6\%) \\
\hline France & $\begin{array}{l}\text { Fine bakery wares } \\
\quad(40 \%)\end{array}$ & Sauces $(12 \%)$ & $\begin{array}{l}\text { Sweetened/flavoured } \\
\text { dairy products }(9 \%)\end{array}$ & $\begin{array}{l}\text { Crackers and addi- } \\
\text { tional bread products } \\
(8 \%)\end{array}$ & Sausages $(6 \%)$ \\
\hline Germany & $\begin{array}{l}\text { Fine bakery wares } \\
(27 \%)\end{array}$ & $\begin{array}{l}\text { Composite dishes } \\
(15 \%)\end{array}$ & Sauces $(12 \%)$ & Sausages $(9 \%)$ & Chocolate (6\%) \\
\hline Greece & $\begin{array}{l}\text { Fine bakery wares } \\
(39 \%)\end{array}$ & $\begin{array}{l}\text { Crackers and addi- } \\
\text { tional bread products } \\
(25 \%)\end{array}$ & Breakfast cereals $(7 \%)$ & Margarines (6\%) & Sauces $(5 \%)$ \\
\hline Hungary & Sausages $(25 \%)$ & Margarines (21\%) & $\begin{array}{l}\text { Fine bakery wares } \\
\quad(13 \%)\end{array}$ & $\begin{array}{l}\text { Crackers and addi- } \\
\text { tional bread products } \\
(6 \%)\end{array}$ & Chocolate (6\%) \\
\hline Ireland & $\begin{array}{l}\text { Fine bakery wares } \\
(25 \%)\end{array}$ & Margarines (12\%) & $\begin{array}{l}\text { Composite dishes } \\
(12 \%)\end{array}$ & Sauces $(11 \%)$ & Breakfast cereals $(9 \%)$ \\
\hline Italy & $\begin{array}{l}\text { Fine bakery wares } \\
(44 \%)\end{array}$ & Sausages (11\%) & $\begin{array}{l}\text { Composite dishes } \\
(8 \%)\end{array}$ & $\begin{array}{l}\text { Water-/dairy-based } \\
\text { desserts (7\%) }\end{array}$ & $\begin{array}{l}\text { Crackers and additional } \\
\text { bread products }(7 \%)\end{array}$ \\
\hline Latvia & $\begin{array}{l}\text { Fine bakery wares } \\
(35 \%)\end{array}$ & Sauces $(14 \%)$ & $\begin{array}{l}\text { Composite dishes } \\
(13 \%)\end{array}$ & Chocolate (11\%) & Sausages $(10 \%)$ \\
\hline The Netherlands & $\begin{array}{l}\text { Fine bakery wares } \\
(20 \%)\end{array}$ & $\begin{array}{l}\text { Composite dishes } \\
(14 \%)\end{array}$ & Sauces $(11 \%)$ & Margarines (11\%) & Chocolate (7\%) \\
\hline Portugal & $\begin{array}{l}\text { Fine bakery wares } \\
(40 \%)\end{array}$ & $\begin{array}{l}\text { Composite dishes } \\
(9 \%)\end{array}$ & Breakfast cereals $(7 \%)$ & $\begin{array}{l}\text { Water-/dairy-based } \\
\text { desserts }(5 \%)\end{array}$ & Sausages $(5 \%)$ \\
\hline Romania & Sausages (18\%) & $\begin{array}{l}\text { Composite dishes } \\
(15 \%)\end{array}$ & $\begin{array}{l}\text { Crackers and addi- } \\
\text { tional bread products } \\
(14 \%)\end{array}$ & $\begin{array}{l}\text { Fine bakery wares } \\
\quad(12 \%)\end{array}$ & Margarines (7\%) \\
\hline Slovenia & $\begin{array}{l}\text { Fine bakery wares } \\
(45 \%)\end{array}$ & Sausages (10\%) & $\begin{array}{l}\text { Crackers and addi- } \\
\text { tional bread products } \\
(10 \%)\end{array}$ & Breakfast cereals $(5 \%)$ & Sauces $(5 \%)$ \\
\hline Spain & $\begin{array}{l}\text { Fine bakery wares } \\
(35 \%)\end{array}$ & $\begin{array}{l}\text { Crackers and addi- } \\
\text { tional bread products } \\
(17 \%)\end{array}$ & Sausages $(9 \%)$ & $\begin{array}{l}\text { Sweetened/flavoured } \\
\text { dairy products }(7 \%)\end{array}$ & Sauces $(5 \%)$ \\
\hline
\end{tabular}


Table 2 (continued)

\begin{tabular}{llllll}
\hline & Top five & & & \\
\cline { 2 - 5 } & 1 & 2 & 3 & Margarines (6\%) & Chocolate (5\%) \\
\hline Sweden & $\begin{array}{c}\text { Composite dishes } \\
(41 \%)\end{array}$ & $\begin{array}{c}\text { Fine bakery wares } \\
(15 \%)\end{array}$ & Sauces (10\%) & Margarines (7\%) & Breakfast cereals (7\%) \\
United Kingdom & $\begin{array}{c}\text { Composite dishes } \\
(29 \%)\end{array}$ & $\begin{array}{c}\text { Fine bakery wares } \\
(16 \%)\end{array}$ & Sauces (13\%) & & \\
\hline
\end{tabular}

Eur Europe

United Kingdom (17.2\%), and Sweden (20.9\%), while the consumption amount of UPDs was similar to that of UPFs in Austria, Belgium, Croatia, Cyprus, Czech Republic, Denmark, Estonia, Hungary, Italy, the Netherlands, Portugal, Romania, and Spain. The share of dietary energy coming from UPFDs for women was also low in Italy (13.8\%) and Romania (15.8\%), while high in the UK (41.3\%) and Sweden $(43.8 \%)$.

\section{Top five of the ultra-processed foods}

Overall, the most consumed UPFs among adults, both men and women, across Europe were fine bakery wares, when expressed in dietary energy share from UPFs (Table 2) and when expressed in daily food consumption amounts, i.e. grams per day (Supplementary Table 2).

The UPFs most frequently ranked among the top five contributors to the dietary energy share of UPFs were: fine bakery wares, sausages, sauces, margarines and composite dishes, followed by crackers and additional bread products, chocolate, breakfast cereals, chocolate, and least frequently ranked were sweetened or flavoured dairy products, water-/dairy-based desserts, and candies and confectionary (Table 2). From this list, fine bakery wares were ranked first in most countries (14 countries for men; 15 countries for women), followed by sausages ( 4 countries for men and 3 countries for women), and margarines and composite dishes ( 2 countries each for both men and women). Comparing men and women, the share of fine bakery wares was lower in men $(25.2 \%$ vs $28.2 \%)$, while that of sausages was higher (15.5\% vs $9.5 \%)$.

The top five of the UPFs consumed by European adults was slightly different when expressing in percentage of daily food consumption amounts as compared to dietary energy shares (Supplementary Table 2). Still, the largest contributor was the group of fine bakery wares (in 9 countries for men, and in 12 countries for women), but closely followed by composite dishes (in 6 countries for men, and in 5 countries for women), and after that by sausages (in 5 countries in men, and in 2 countries in women), and margarines (Denmark in both men and women), and also sweetened or flavoured dairy products (Finland for men and Finland and Estonia for women). Besides, these sweetened or flavoured dairy products were also identified as the top five contributor in the food consumption share of UPFs in 13 countries for men and 18 countries for women (while only in Finland, France and Spain when considering dietary energy share), most often at the expense of chocolate or margarine.

\section{Top three of ultra-processed drinks}

Overall, the most consumed UPDs among adults across Europe were soft drinks, when expressed both in dietary energy share from UPDs (Table 3), or in daily food consumption amounts (Supplementary Table 3).

Soft drinks ranked as the main contributor to the dietary energy share of UPDs for men in all European countries included, while for women also fruit juices not $100 \%$ from named source (3 countries), sweetened or flavoured milk (2 countries), cocoa beverages and soups (1 country each) were identified as main contributors (Table 3). Expressed in daily food consumption amounts, soft drinks consistently ranked first in all countries for both men and women (Supplementary Table 3 ). In addition, diet soft drinks were observed in the top three of UPDs in nine countries for both men and women.

\section{Time-changes in ultra-processed foods and drinks}

Figure 1 shows the time-changes in the dietary energy share from UPFDs for countries with available data. In six countries, a decrease in the share of dietary energy from UPFDs was observed, with generally a slightly larger decrease for women than for men, and the largest decrease for Austria (13\% for men vs $15 \%$ for women), Belgium (7\% vs $9 \%$ ), and Latvia (6\% vs $11 \%$ ). No clear time-changes were observed for Ireland and France, and Finnish men (less than 2\%), while the share of dietary energy from UPFDs was higher for the most recent survey in the UK $(9 \%$ and $8 \%$ for men and women, respectively), Spain ( $4 \%$ for both men and women) and Finland (4\% for women only). 
Table 3 The top three of the ultra-processed drinks consumed by European adults, as available by EFSA, ordered alphabetically and stratified by sex, expressed in percentage of daily energy intake from ultra-processed drinks

\begin{tabular}{|c|c|c|c|}
\hline & \multicolumn{3}{|l|}{ Top three } \\
\hline & 1 & 2 & 3 \\
\hline Eur-22 countries & Soft drinks $(5.0 \%)$ & $\begin{array}{l}\text { Fruit/vegetables juices not } 100 \% \\
\text { from named source }(1.4 \%)\end{array}$ & Sweetened/flavoured milk (1.1\%) \\
\hline \multicolumn{4}{|l|}{ Men } \\
\hline Eur-22 countries & Soft drinks $(5.7 \%)$ & $\begin{array}{l}\text { Fruit/vegetables juices not } 100 \% \\
\text { from named source }(1.3 \%)\end{array}$ & Sweetened/flavoured milk $(0.9 \%)$ \\
\hline Austria & Soft drinks (9\%) & $\begin{array}{l}\text { Fruit/vegetables juices not } 100 \% \\
\text { from named source }(2 \%)\end{array}$ & Sweetened/flavoured milk (1\%) \\
\hline Belgium & Soft drinks $(8 \%)$ & Sweetened/flavoured milk (1\%) & Milk imitates $(0.4 \%)$ \\
\hline Croatia & Soft drinks $(6 \%)$ & $\begin{array}{l}\text { Fruit/vegetables juices not } 100 \% \\
\text { from named source }(6 \%)\end{array}$ & Cocoa beverages $(0.3 \%)$ \\
\hline Cyprus & Soft drinks (10\%) & $\begin{array}{l}\text { Fruit/vegetables juices not } 100 \% \\
\text { from named source }(1 \%)\end{array}$ & Soups $(0.4 \%)$ \\
\hline Czech Republic & Soft drinks (7\%) & $\begin{array}{l}\text { Fruit/vegetables juices not } 100 \% \\
\text { from named source }(1 \%)\end{array}$ & Cocoa beverages $(0.3 \%)$ \\
\hline Denmark & Soft drinks $(12 \%)$ & $\begin{array}{l}\text { Fruit/vegetables juices not } 100 \% \\
\text { from named source }(2 \%)\end{array}$ & Sweetened/flavoured milk ( $2 \%)$ \\
\hline Estonia & Soft drinks (4\%) & $\begin{array}{l}\text { Fruit/vegetables juices not } 100 \% \\
\text { from named source }(2 \%)\end{array}$ & Sweetened/flavoured milk (1\%) \\
\hline Finland & Soft drinks $(2 \%)$ & Cocoa beverages (1\%) & Sweetened/flavoured milk $(0.5 \%)$ \\
\hline France & Soft drinks (4\%) & Cocoa beverages (1\%) & $\begin{array}{l}\text { Fruit/vegetables juices not } 100 \% \text { from named } \\
\text { source }(1 \%)\end{array}$ \\
\hline Germany & Soft drinks (4\%) & Soups (3\%) & $\begin{array}{l}\text { Fruit/vegetables juices not } 100 \% \text { from named } \\
\text { source }(2 \%)\end{array}$ \\
\hline Greece & Soft drinks (3\%) & $\begin{array}{l}\text { Fruit/vegetables juices not } 100 \% \\
\text { from named source }(2 \%)\end{array}$ & Sweetened/flavoured milk $(0.2 \%)$ \\
\hline Hungary & Soft drinks $(6 \%)$ & Sweetened/flavoured milk (6\%) & Cocoa beverages (1\%) \\
\hline Ireland & Soft drinks (5\%) & $\begin{array}{l}\text { Fruit/vegetables juices not } 100 \% \\
\text { from named source }(1 \%)\end{array}$ & Sweetened/flavoured milk $(0.4 \%)$ \\
\hline Italy & Soft drinks (3\%) & $\begin{array}{l}\text { Fruit/vegetables juices not } 100 \% \\
\text { from named source (3\%) }\end{array}$ & Cocoa beverages (1\%) \\
\hline Latvia & Soft drinks (2\%) & $\begin{array}{l}\text { Fruit/vegetables juices not } 100 \% \\
\text { from named source }(1 \%)\end{array}$ & Cocoa beverages $(0 \%)$ \\
\hline The Netherlands & Soft drinks $(6 \%)$ & Sweetened/flavoured milk (2\%) & $\begin{array}{l}\text { Fruit/vegetables juices not } 100 \% \text { from named } \\
\text { source }(1 \%)\end{array}$ \\
\hline Portugal & Soft drinks (10\%) & Sweetened/flavoured milk (4\%) & $\begin{array}{l}\text { Fruit/vegetables juices not } 100 \% \text { from named } \\
\text { source }(3 \%)\end{array}$ \\
\hline Romania & Soft drinks $(8 \%)$ & Soups $(4 \%)$ & Cocoa beverages $(0.5 \%)$ \\
\hline Slovenia & Soft drinks (3\%) & Cocoa beverages (1\%) & $\begin{array}{l}\text { Fruit/vegetables juices not } 100 \% \text { from named } \\
\text { source }(1 \%)\end{array}$ \\
\hline Spain & Soft drinks (4\%) & Milk imitates (1\%) & Cocoa beverages (1\%) \\
\hline Sweden & Soft drinks (3\%) & Soups $(2 \%)$ & $\begin{array}{l}\text { Fruit/vegetables juices not } 100 \% \text { from named } \\
\text { source }(1 \%)\end{array}$ \\
\hline United Kingdom & Soft drinks (5\%) & Soups (1\%) & Milkshakes $(0.4 \%)$ \\
\hline \multicolumn{4}{|l|}{ Women } \\
\hline Eur-22 countries & Soft drinks $(4.5 \%)$ & $\begin{array}{l}\text { Fruit/vegetables juices not } 100 \% \\
\text { from named source }(1.5 \%)\end{array}$ & Sweetened/flavoured milk (1.3\%) \\
\hline Austria & Soft drinks $(6 \%)$ & $\begin{array}{l}\text { Fruit/vegetables juices not } 100 \% \\
\text { from named source }(2 \%)\end{array}$ & Sweetened/flavoured milk (1\%) \\
\hline Belgium & Soft drinks (6\%) & Sweetened/flavoured milk (1\%) & Milk imitates (1\%) \\
\hline Croatia & $\begin{array}{l}\text { Fruit/vegetables juices not } 100 \% \\
\text { from named source }(6 \%)\end{array}$ & Soft drinks (6\%) & Cocoa beverages (1\%) \\
\hline
\end{tabular}


Table 3 (continued)

\begin{tabular}{|c|c|c|c|}
\hline & \multicolumn{3}{|l|}{ Top three } \\
\hline & 1 & 2 & 3 \\
\hline Cyprus & Soft drinks $(6 \%)$ & $\begin{array}{l}\text { Fruit/vegetables juices not } 100 \% \\
\text { from named source }(1 \%)\end{array}$ & Soups (1\%) \\
\hline Czech Republic & Soft drinks (7\%) & $\begin{array}{l}\text { Fruit/vegetables juices not } 100 \% \\
\text { from named source }(3 \%)\end{array}$ & Cocoa beverages $(0.3 \%)$ \\
\hline Denmark & Soft drinks (14\%) & Sweetened/flavoured milk (3\%) & $\begin{array}{l}\text { Fruit/vegetables juices not } 100 \% \text { from named } \\
\text { source }(2 \%)\end{array}$ \\
\hline Estonia & $\begin{array}{l}\text { Fruit/vegetables juices not } 100 \% \\
\text { from named source }(2 \%)\end{array}$ & Soft drinks ( $2 \%)$ & Sweetened/flavoured milk ( $2 \%$ ) \\
\hline Finland & Cocoa beverages (1\%) & Soft drinks (1\%) & Sweetened/flavoured milk (1\%) \\
\hline France & Soft drinks (4\%) & Cocoa beverages $(1 \%)$ & $\begin{array}{l}\text { Fruit/vegetables juices not } 100 \% \text { from named } \\
\text { source }(1 \%)\end{array}$ \\
\hline Germany & Soups $(3 \%)$ & Soft drinks ( $2 \%)$ & $\begin{array}{l}\text { Fruit/vegetables juices not } 100 \% \text { from named } \\
\text { source }(2 \%)\end{array}$ \\
\hline Greece & Soft drinks (2\%) & $\begin{array}{l}\text { Fruit/vegetables juices not } 100 \% \\
\text { from named source }(1 \%)\end{array}$ & Cocoa beverages $(0.3 \%)$ \\
\hline Hungary & Sweetened/flavoured milk (7\%) & Soft drinks (4\%) & Cocoa beverages (1\%) \\
\hline Ireland & Soft drinks (4\%) & $\begin{array}{l}\text { Fruit/vegetables juices not } 100 \% \\
\text { from named source (1\%) }\end{array}$ & Sweetened/flavoured milk (0.5\%) \\
\hline Italy & $\begin{array}{l}\text { Fruit/vegetables juices not } 100 \% \\
\text { from named source }(3 \%)\end{array}$ & Soft drinks (2\%) & Cocoa beverages (1\%) \\
\hline Latvia & Soft drinks (1\%) & $\begin{array}{l}\text { Fruit/vegetables juices not } 100 \% \\
\text { from named source }(1 \%)\end{array}$ & Cocoa beverages $(0.2 \%)$ \\
\hline The Netherlands & Soft drinks (5\%) & Sweetened/flavoured milk (3\%) & $\begin{array}{l}\text { Fruit/vegetables juices not } 100 \% \text { from named } \\
\text { source }(2 \%)\end{array}$ \\
\hline Portugal & Sweetened/flavoured milk (6\%) & Soft drinks (5\%) & $\begin{array}{l}\text { Fruit/vegetables juices not } 100 \% \text { from named } \\
\text { source }(2 \%)\end{array}$ \\
\hline Romania & Soft drinks $(8 \%)$ & Soups $(5 \%)$ & Cocoa beverages (1\%) \\
\hline Slovenia & Soft drinks (2\%) & Sweetened/flavoured milk (1\%) & $\begin{array}{l}\text { Fruit/vegetables juices not } 100 \% \text { from named } \\
\text { source }(1 \%)\end{array}$ \\
\hline Spain & Soft drinks (4\%) & Milk imitates (2\%) & Cocoa beverages $(1 \%)$ \\
\hline Sweden & Soft drinks ( $2 \%)$ & Soups (2\%) & $\begin{array}{l}\text { Fruit/vegetables juices not } 100 \% \text { from named } \\
\text { source }(1 \%)\end{array}$ \\
\hline United Kingdom & Soft drinks (5\%) & Soups $(2 \%)$ & Cocoa beverages $(0.4 \%)$ \\
\hline
\end{tabular}

Eur Europe

\section{Are countries with a higher consumption of ultra-processed foods and drinks associates of a lower consumption of dietary fibre and a higher consumption of total sugar intake?}

No associations were found for consumptionof UPFDs and fibre intake (Fig. 2), while countries with diets high in total sugar intake were also the countries with a higher share of dietary energy from UPFs and UPDs, as seen for both men and women (Fig. 3). Weak-moderate correlations were only seen for the energy percentage of UPFs and total sugar intake ( $r=0.57, p$ value $=0.032$ for men; and $r=0.53$, $p$ value $=0.061$ for women $)$.

\section{Do countries with a higher consumption of ultra-processed foods and drinks also have a higher burden of high BMI?}

Figure 4 shows the bivariate analyses for the potential association for the burden of high BMI and energy percentage of UPFDs, stratified by sex and dietary assessment method, as visualised by scatterplots. Countries' burden of high BMI was not observed to increase across increasing percentages of energy from UPFDs, and no correlations were observed. 
Fig. 1 Time-changes of the dietary energy share from ultraprocessed foods and drinks in the European adult population, with recurrent dietary survey intake data available by EFSA, stratified by sex and method of dietary assessment. Legend: 24-h recalls;

Food records. AT Austria, $B E$ Belgium, $D K$ Denmark, $E N \%$ energy percentages, $E S$ Spain, FI Finland, FR France, $L V$ Latvia, $I E$ Ireland, $N L$ the Netherlands, $S E$ Sweden, $U K$ United Kingdom, UPFD ultraprocessed foods and drinks
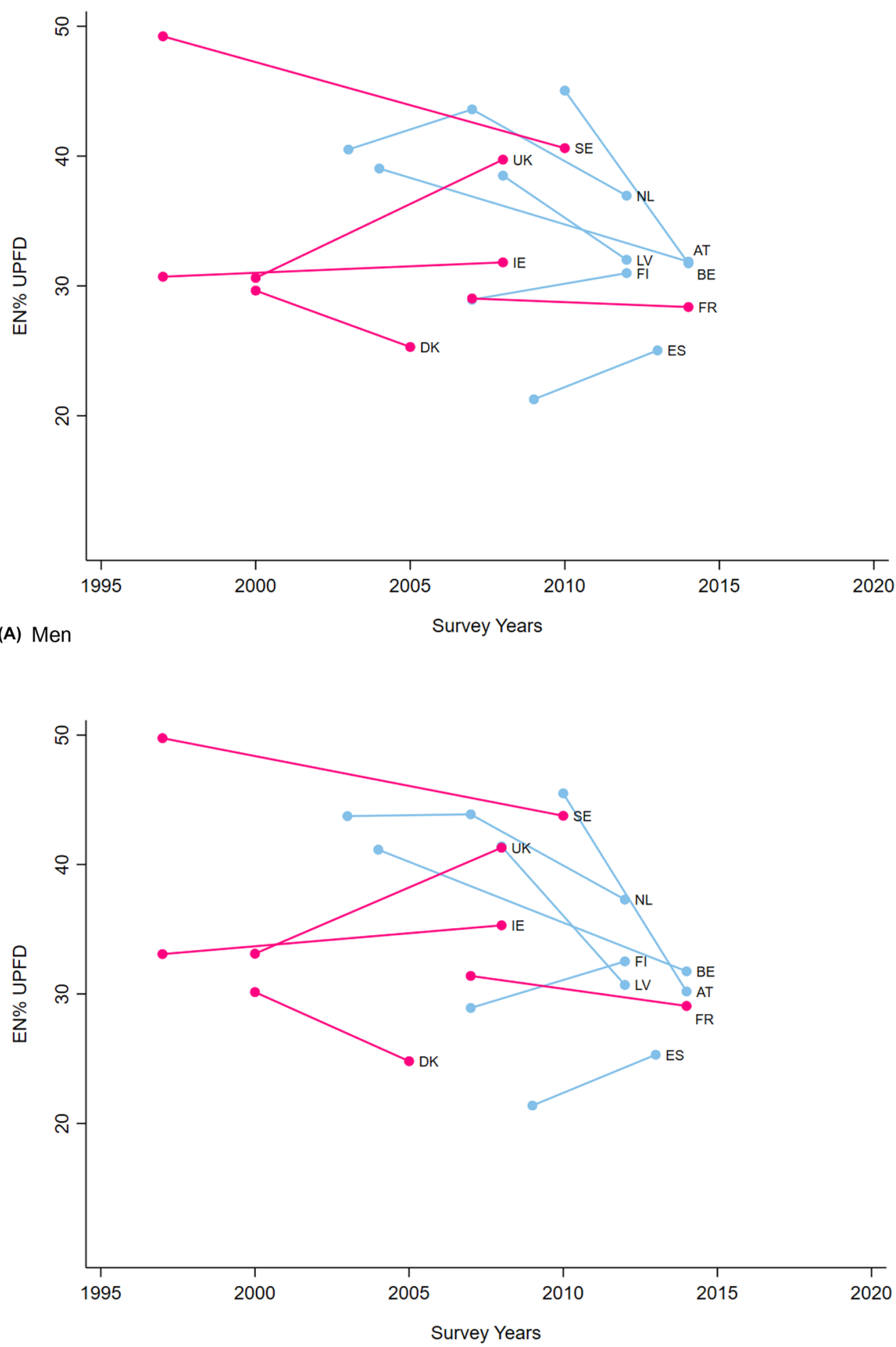

(B) Women

\section{Discussion}

Using data on country-specific dietary intakes, the present study found that the share of dietary energy originating from UPFDs consumption varied markedly across the 22 European countries included in the analysis, ranging from 14 to
$44 \%$ with the lowest share for Italy, Romania and Hungary, and the highest for the Netherlands, Germany, the UK and Sweden. Fine bakery wares and soft drinks were the main contributor to the dietary energy share of UPFs and UPDs, respectively. 

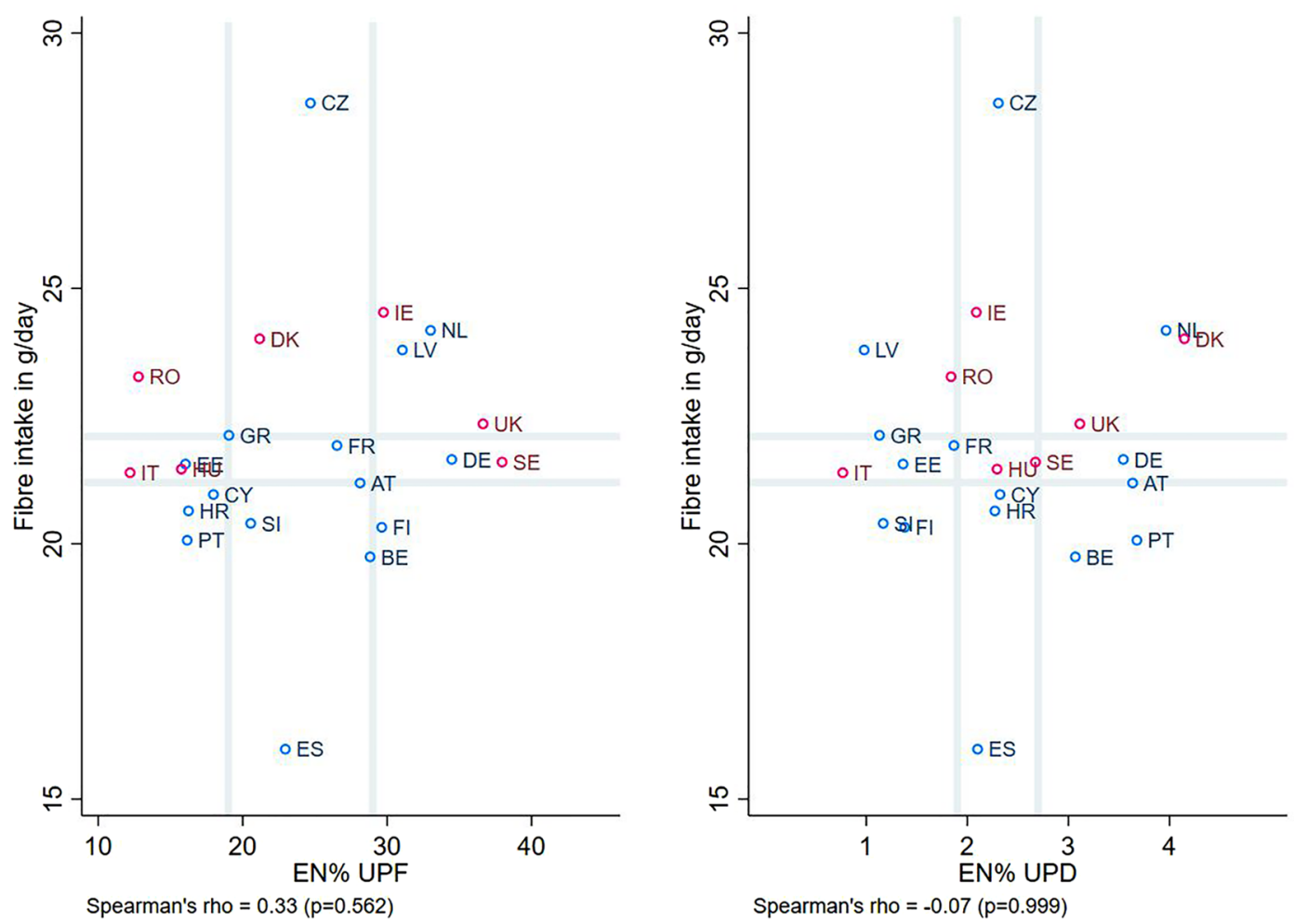

(A) Men
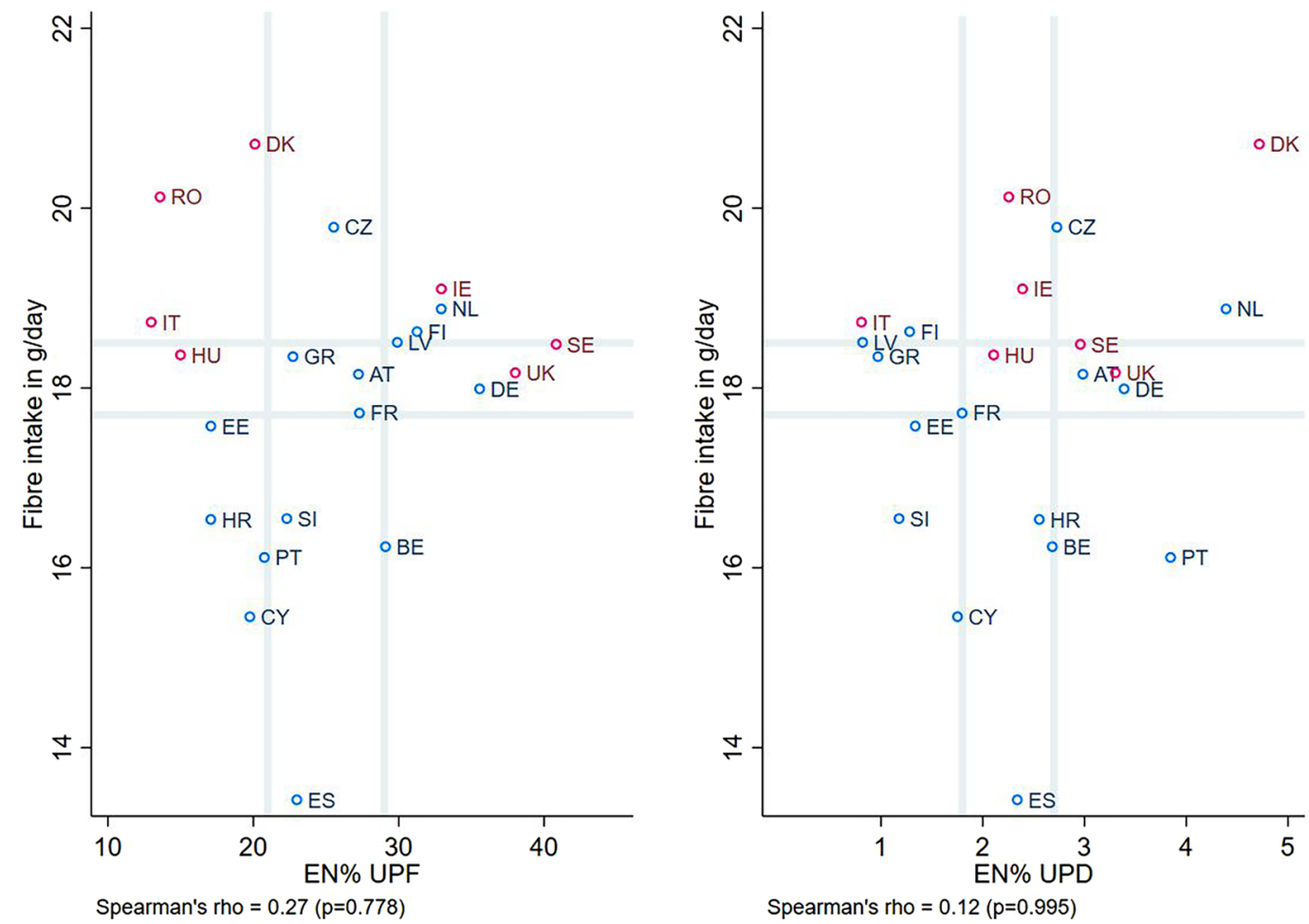

(B) Women 
4Fig. 2 Scatterplot of fibre intake (in grams per day) against the percentage of energy coming from ultra-processed foods and drinks, stratified sex, and dietary assessment method. Legend: o 24-hR; o Food records. Grey vertical and horizontal gridlines indicate tertiles dividing lines for the measures: the share of dietary energy from UPFs (at 19.0 and $28.8 \mathrm{EN} \%$ for men, and at 20.7 and $29.1 \mathrm{EN} \%$ for women) and UPDs (at 1.9 and $2.7 \mathrm{EN} \%$ for men, and at 1.8 and 2.7 EN\% for women), and fibre intake (at 21.2 and $22.1 \mathrm{~g} /$ day for men, and at 17.7 and $18.5 \mathrm{~g} /$ day for women). $A T$ Austria, $B E$ Belgium, $C Y$ Cyprus, $C Z$ Czech Republic, $D E$ Germany, $D K$ Denmark, $E E$ Estonia, EN\% energy percentage, ES Spain, FI Finland, FR France, GR Greece, $H R$ Croatia, $H U$ Hungary, IE Ireland, IT Italy, $L V$ Latvia, $N L$ the Netherlands, $P T$ Portugal, $R O$ Romania, SE Sweden, SI Slovenia, $U K$ United Kingdom, $U P D$ ultra-processed drinks, $U P F$ ultra-processed foods

Our results on the dietary energy share of UPFDs are in the range as those reported earlier using food purchase data across Europe [13]. However, the levels observed in the present study differed from those reported in previous studies that also used individual-level national dietary data analysed at the individual level. Our estimates were slightly higher for Belgium [14], but slightly lower for France [15] and Portugal [16] and up to around 20\% lower for the UK for surveys (years) similar to the ones included in the present analyses, and also much lower as compared to earliest results (dated from 1995 to 2000) from middle-aged adults from ten European countries participating in the European Prospective Investigation into Cancer and Nutrition (EPIC) that reported a range between 60 and $80 \%$ [30]. These discrepancies may be due to differential use of the NOVA classification and its application depending on the variety of foods captured in the dietary database selected for the estimation of UPFDs [31, 32]. In our study, the use of FoodEx2 did not allow to disentangle breads withhout extra ingredients, except nuts and seeds, that are processed, from the industrially produced ones, that are ultra-processed, contrary to what is done in reports from individual countries where information on production and/or packaging is available $[15,16,18]$, hence likely resulting in an underestimation in our study. The exclusion of distilled alcoholic beverages from our definition of UPFDs might also marginally underestimate our results, since the average share of total energy intake from these beverages was $0.42 \%$ (with a range from 0 to $2.5 \%$ ) for the European countries included, representing when included in UPFDs an average contribution of 1.6 percent (data not shown).

Comprehensive understanding of the contribution of UPFDs to dietary quality would require describing consumption in amounts of grams and energy intakes, rather than the dietary energy share of UPFDs alone, as often done in the literature. This is clearly illustrated by the contribution of drinks to UPFDs consumption that is between 16 and $64 \%$ of the total UPFDs amount consumed and only between 3 and 19\% of the energy intake from UPFDs. Therefore, describing food consumption solely in energy percentages functionally disregards the role of low-/non-energy bearing foods, such as diet soft drinks, on dietary quality in terms of the extent and purpose of industrial processing, as assessed by the NOVA classification.

Most prior studies found that higher consumption of UPFDs were associated with a higher BMI, as reported for example by a recent narrative review covering study designs from three ecological, ten cross-sectional and two prospective cohort studies, and one randomised controlled crossover trial [7]. Our results, however, do not fully support these previous observations. The ecological design of our analysis is mostly relevant for a descriptive comparison across Europe and does not account for socio-demographic drivers of food choices, such as socio-economic status, or other determinants of high BMI, such as physical inactivity. Thus, with population being the unit of analysis, caution must be applied, as findings cannot be extrapolated to high-risk individuals within countries because of ecological fallacy. Nevertheless, the consumption of UPFDs that are often of low-nutritional quality (i.e. energy-dense, lowfibre, nutrient-depleted, and/or high-sodium, and high-added sugars) would in principle hinder adherence to a healthy, optimal, diet. Relating to dietary quality of the foods consumed, it is not surprising that the intake of total sugars is higher with increasing intakes of UPFDs [18, 29, 33-35] because they are among the major sources of added sugar in the diet [5]. Our study could confirm also this tendency for total sugars, but not the anticipated trend in the opposite direction for dietary fibre, i.e. a nutrient closely linked with unprocessed or minimally processed foods, like whole grains, fruits, legumes and vegetables. Yet, this null-finding emphasises that UPFDs might be as crucial in nutrient provision as unprocessed or minimally processed foods [36, 37], also related to the fact that UPFDs account for a large part of European diets and contributes on average one-sixth of total daily dietary fibre intake (data not shown). Moreover, at the food level, when compared to the foods not ultra-processed, the UPFDs often contain more carbohydrates of low-quality, reflected utmost in the levels of added sugar (3.4\% for foods not ultra-processed vs $19.3 \%$ for UPFDs) rather than dietary 

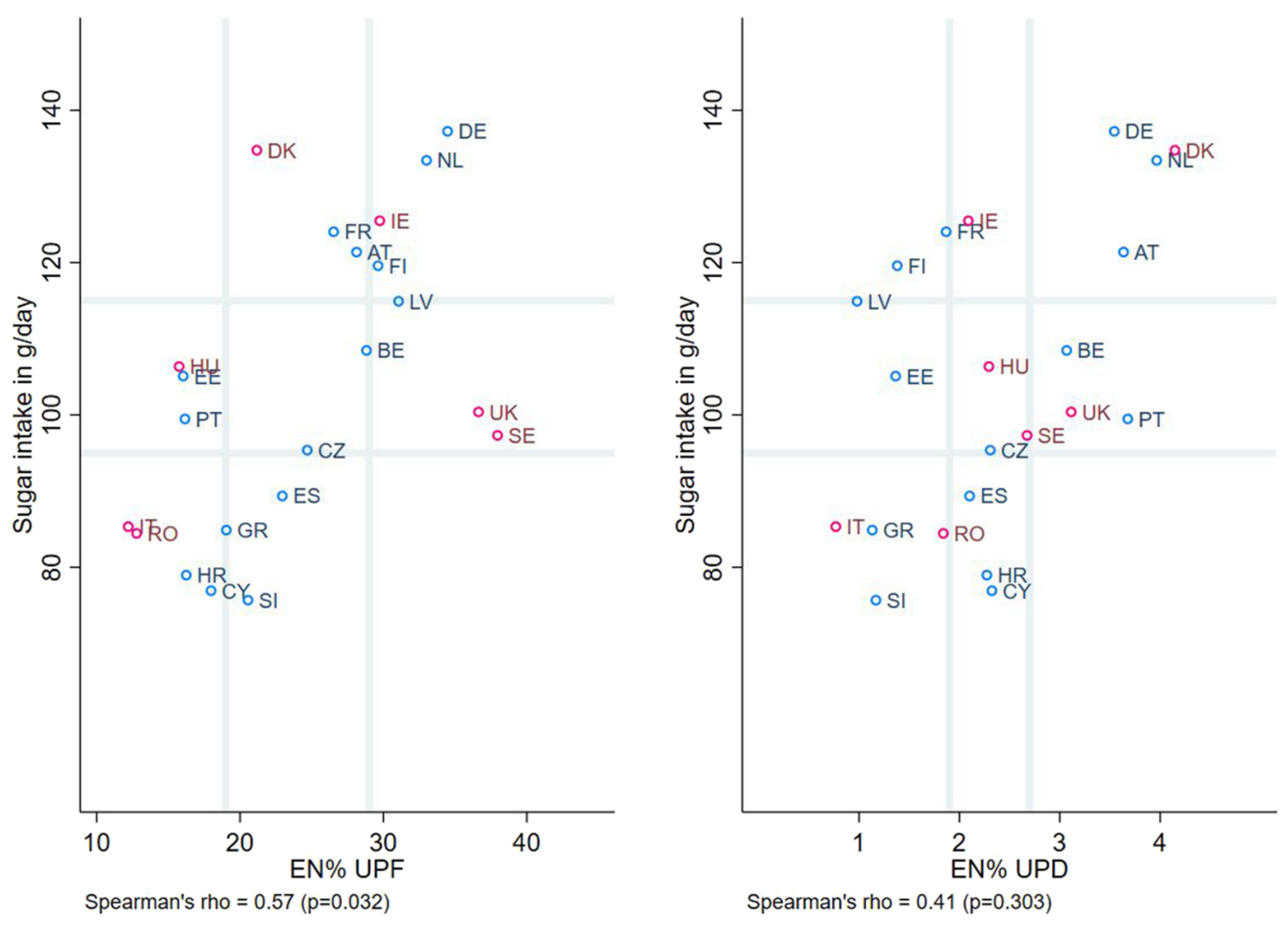

(A) Men
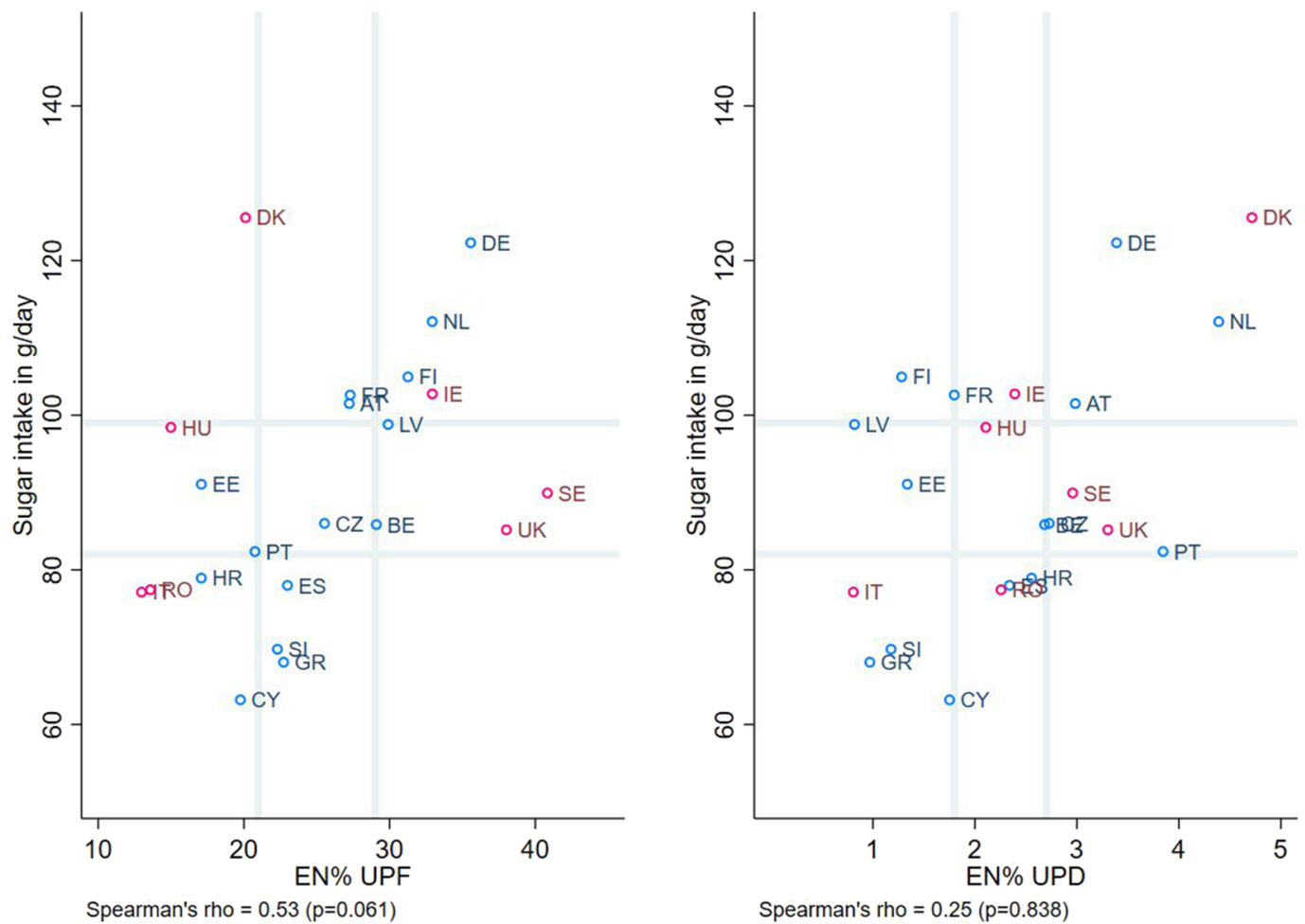

(B) Women 
4Fig. 3 Scatterplot of total sugar intake (in grams per day) against the percentage of energy coming from ultra-processed foods and drinks, stratified by sex, and dietary assessment method. Legend: o 24-hR; o Food records. Grey vertical and horizontal gridlines indicate tertiles dividing lines for the measures: the share of dietary energy from UPFs (at 19.0 and $28.8 \mathrm{EN} \%$ for men, and at 20.7 and $29.1 \mathrm{EN} \%$ for women) and UPDs (at 1.9 and $2.7 \mathrm{EN} \%$ for men, and at 1.8 and 2.7 EN\% for women), and sugar intake (at 95.4 and $115.0 \mathrm{~g} /$ day for men, and at 82.4 and $98.8 \mathrm{~g} /$ day for women). $A T$ Austria, $B E$ Belgium, $C Y$ Cyprus, $C Z$ Czech Republic, $D E$ Germany, $D K$ Denmark, $E E$ Estonia, EN\% energy percentage, ES Spain, FI Finland, FR France, GR Greece, $H R$ Croatia, $H U$ Hungary, IE Ireland, IT Italy, $L V$ Latvia, $N L$ the Netherlands, $P T$ Portugal, $R O$ Romania, SE Sweden, SI Slovenia, $U K$ United Kingdom, $U P D$ ultra-processed drinks, $U P F$ ultra-processed foods

fibre $(0.87 \mathrm{~g} / 100 \mathrm{kcal}$ vs $0.67 \mathrm{~g} / 100 \mathrm{kcal})$, as reported by the nutrient profiles of the foods consumed by USA youths [38].

As expected, the share of dietary energy from UPFDs across Europe is lower than those reported for other Western countries such as the USA [33, 39, 40] and Canada [35], reporting intakes of up to around $60 \%$ of the energy intake. Still, our study confirms the existence of a geographical gradient for the contribution of UPFDs to European diets, i.e. they are highly dominating the diets of Western European countries, but not yet those of countries located in Central, the East and the South of Europe, consistent with prior studies (one dated from 1995 to 2000 [30] and two using purchase data $[12,13])$.

Sales of UPFDs have on average slightly decreased over the period 2002-2016 for Western Europe, notwithstanding remaining high, while those of Central and Eastern Europe increased reflecting the move towards more processed diets [12]. Comparison of time-changes in UPFDs sales of individual European countries and our observations for the countries with recurrent food consumption surveys showed disagreements, pinpointing the challenges involved in the different methods of dietary data collection and analyses [41]. However, sales and purchase data for food and drinks can only be regarded as a proxy of food consumption data, because of the food practices between purchase and final consumption, including food preparation, distribution among household members and waste and/or in the accuracy of reporting dietary data.

The present study provides further support for the utilization of comprehensive nutritional databases in epidemiological studies to address current dietary concerns as well as the diet-health relationships. Cross-country comparison of dietary data, collected at the individual-level, is, however, challenged by the conduct of the dietary surveys related to the survey characteristics and data collection methods that may influence comparability of the results. To overcome this limitation in the future, the EFSA has since 2014 launched European Union Menu Project, i.e. an Europe-wide initiative aimed at a standardised collection of accurate, harmonised and detailed food consumption data across Member States [42]. For the time being, the present analyses were based on dietary data collected by means of at least two days of food records or 24-h recalls, since both are reporting on the foods and amounts that are actually consumed by an individual on specific days, hereby allowing greater specificity for describing foods, although its limitations of being resource-intensive, and subject to misreporting [43].

Expressing food consumption data as energy percentages might potentially partly account for the extraneous variation in dietary estimates due to measurement error. However, the use of the Dutch food composition database NEVO to calculate dietary composition, including the intake of energy, fibre and sugar, of the different national diets across Europe might limit the accuracy of our estimates, and decrease variability across countries. This is because of the lack of data to account for country-specific food composition, but also the foods and composite dishes available in the different countries are not necessarily produced/prepared in the same manner despite globalisation of dietary patterns. Nevertheless, the use of the same food composition database to estimate dietary composition increases cross-country comparability by cancelling out any potential systematic bias that may exist in country-specific databases and likely to vary in magnitude and direction. Our analyses, therefore, allow for direct comparison of the nutrient intakes, as any differences in nutrient intakes exclusively originate from the composition of diet instead of a mingling of food and dietary composition.

Another attempt for dietary data harmonisation of this study related to the use of a common food classification system, i.e. FoodEx2, to report food consumption consistently across countries, and subsequently calculate intakes according to the NOVA classification and food subgroups uniformly. Still, results would be highly relying on the coding-details of FoodEx2, and in all probability influence our estimate of UPFDs consumption, as previously discussed. Any possible discrepancy with previous reports may be due to the report of alcoholic beverages that were not classified and/or the composite dishes that were all classified as ultra-processed without considering any classification according to the NOVA classification for the disaggregated ingredients ofhome-prepared dishes, as often done when individual-level data are available. The present study applying the EFSA nutritional database constructed from individual-level dietary data highlights the importance of using a common food classification system and the same food composition database for aligning dietary data from the consumer domain, and hereby enabling cross-country comparisons of the diet, from the consumption of foods to the intake of nutrients. 

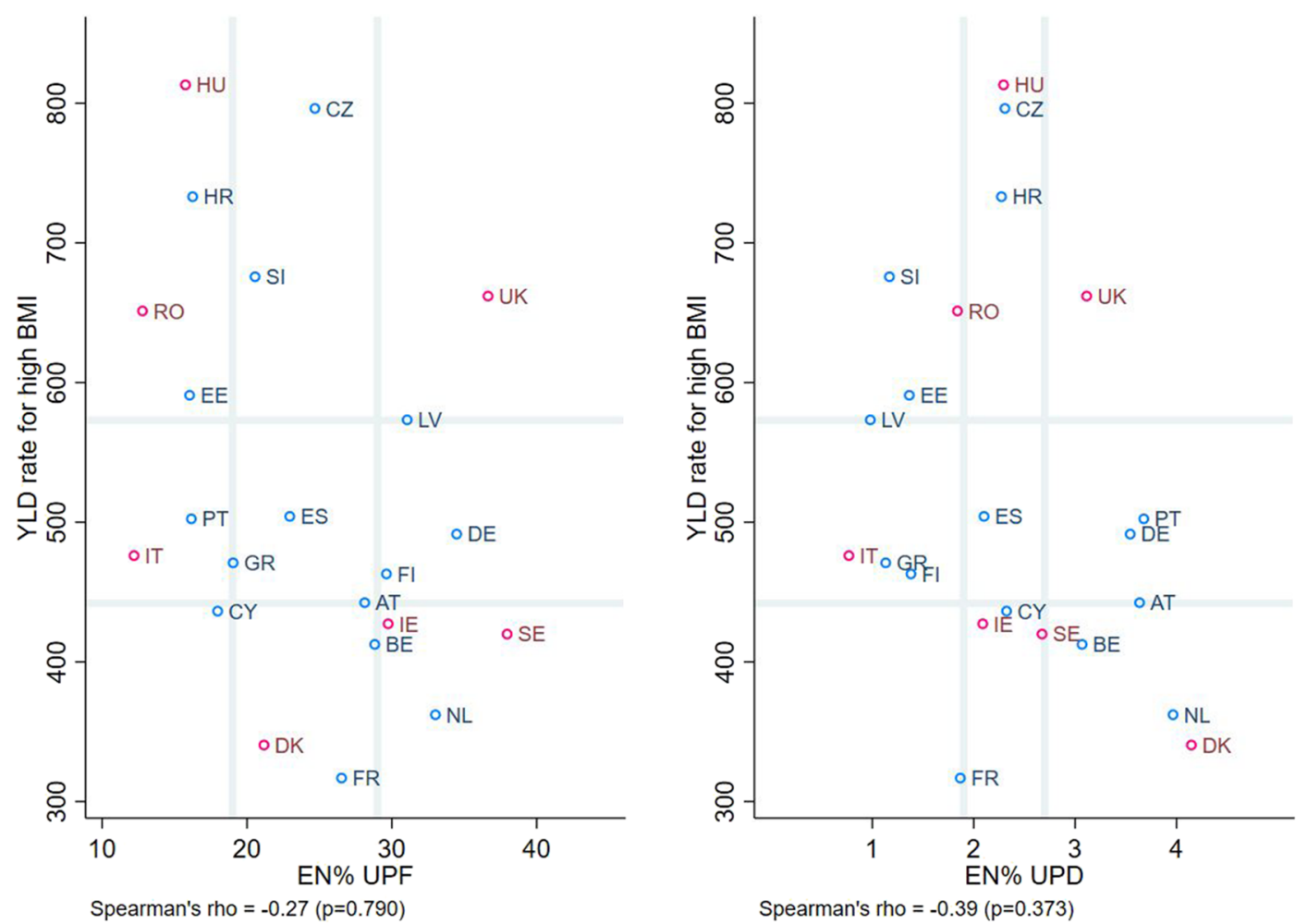

(A) Men
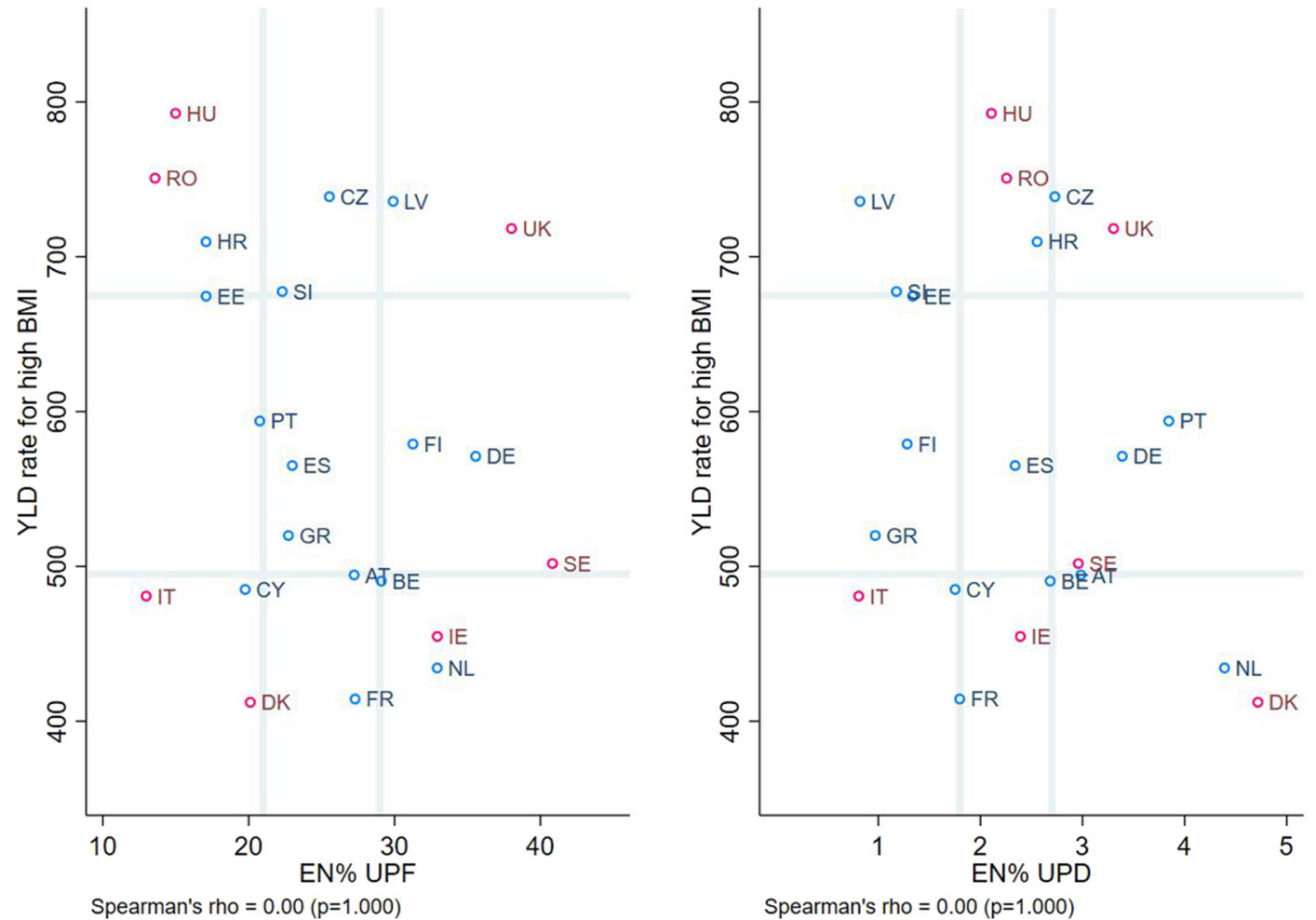

(B) Women 
४Fig. 4 Scatterplot of YLD rate for high BMI against the percentage of energy derived from ultra-processed foods and drinks, stratified by sex, and dietary assessment method. Legend: o 24-hR; o Food records. Grey vertical and horizontal gridlines indicate tertiles dividing lines for the measures: the share of dietary energy from UPFs (at 19.0 and $28.8 \mathrm{EN} \%$ for men, and at 20.7 and $29.1 \mathrm{EN} \%$ for women) and UPDs (at 1.9 and $2.7 \mathrm{EN} \%$ for men, and at 1.8 and $2.7 \mathrm{EN} \%$ for women), and YLD for high BMI per 100,000 (at 443 and 573 for men, and at 495 and 675 for women). $A T$ Austria, $B E$ Belgium, $C Y$ Cyprus, $C Z$ Czech Republic, $D E$ Germany, $D K$ Denmark, $E E$ Estonia, EN\% energy percentage, ES Spain, FI Finland, FR France, GR Greece, $H R$ Croatia, $H U$ Hungary, IE Ireland, IT Italy, $L V$ Latvia, $N L$ the Netherlands, $P T$ Portugal, $R O$ Romania, SE Sweden, SI Slovenia, $U K$ United Kingdom, $U P D$ ultra-processed drinks, $U P F$ ultra-processed foods, $Y L D$ Years Lived with Disability, in age-standardised rates per 100,000

\section{Conclusion}

This study aimed at describing UPFDs consumption across Europe found considerable variation in the proportion of amounts consumed according to energy intakes by European adults, irrespective of sex, while similar main contributors to the UPFDs consumption were identified across countries and sex. Population-level consumption of UPFDs did not appear to be associated with a country-level burden of high BMI, despite being related to higher total sugar intake.

Supplementary Information The online version contains supplementary material available at https://doi.org/10.1007/s00394-021-02733-7.

Author contributions JP and EM conceptualised and designed the study, identified the relevant data sources, and retrieved the data. EM performed the statistical analyses. EM, CC, and JP wrote the manuscript, and all authors approved the submitted version.

Funding Research supported by the Research Foundation of Flanders (FWO), Grant G0C2520N.

Availability of data and material Publicly available datasets were analysed in this study. These data can be found here: https://data. europa.eu/data/datasets/the-efsa-comprehensive-european-food-consu mption-database?locale=en; https://nevo-online.rivm.nl/; http://ghdx. healthdata.org/gbd-results-tool. In addition, a copy of the Dutch Food Consumption Survey (FCS) 2012-2016 was requested via https://www. wateetnederland.nl/publicaties-en-datasets/datasets, and was used to initiate the linkage between FoodEx 2 and food composition data, i.e. NEVO, the Dutch Food Composition Table.

Code availability STATA DO-files are available on request.

\section{Declarations}

Conflict of interest The authors declare that the research was conducted in the absence of any commercial or financial relationships that could be construed as a potential conflict of interest.

Ethical approval Not applicable.
Open Access This article is licensed under a Creative Commons Attribution 4.0 International License, which permits use, sharing, adaptation, distribution and reproduction in any medium or format, as long as you give appropriate credit to the original author(s) and the source, provide a link to the Creative Commons licence, and indicate if changes were made. The images or other third party material in this article are included in the article's Creative Commons licence, unless indicated otherwise in a credit line to the material. If material is not included in the article's Creative Commons licence and your intended use is not permitted by statutory regulation or exceeds the permitted use, you will need to obtain permission directly from the copyright holder. To view a copy of this licence, visit http://creativecommons.org/licenses/by/4.0/.

\section{References}

1. Monteiro CA (2009) Nutrition and health. The issue is not food, nor nutrients, so much as processing. Public Health Nutr 12(5):729-731. https://doi.org/10.1017/S1368980009005291

2. Monteiro CA, Cannon G, Lawrence M, Costa Louzada ML, Pereira Machado P (2019) Ultra-processed foods, diet quality and health using the NOVA classification system. FAO, Rome

3. Monteiro CA, Cannon G, Moubarac JC, Levy RB, Louzada MLC, Jaime PC (2018) The UN Decade of Nutrition, the NOVA food classification and the trouble with ultra-processing. Public Health Nutr 21(1):5-17. https://doi.org/10.1017/s1368980017000234

4. Monteiro CA, Levy RB, Claro RM, de Castro IRR, Cannon G (2010) Increasing consumption of ultra-processed foods and likely impact on human health: evidence from Brazil. Public Health Nutr 14(1):5-13. https://doi.org/10.1017/S1368980010003241

5. Monteiro CA, Cannon G, Levy RB, Moubarac J-C, Louzada MLC, Rauber F, Khandpur N, Cediel G, Neri D, Martinez-Steele E, Baraldi LG, Jaime PC (2019) Ultra-processed foods: what they are and how to identify them. Public Health Nutr 22(5):936-941. https://doi.org/10.1017/S1368980018003762

6. Ludwig DS (2011) Technology, diet, and the burden of chronic disease. JAMA 305(13):1352-1353. https://doi.org/10.1001/jama. 2011.380

7. Elizabeth L, Machado P, Zinöcker M, Baker P, Lawrence M (2020) Ultra-processed foods and health outcomes: a narrative review. Nutrients 12(7):1955. https://doi.org/10.3390/nu12071955

8. Chen X, Zhang Z, Yang H, Qiu P, Wang H, Wang F, Zhao Q, Fang J, Nie J (2020) Consumption of ultra-processed foods and health outcomes: a systematic review of epidemiological studies. Nutr J 19(1):86. https://doi.org/10.1186/s12937-020-00604-1

9. Srour B, Fezeu LK, Kesse-Guyot E, Allès B, Debras C, DruesnePecollo N, Chazelas E, Deschasaux M, Hercberg S, Galan P, Monteiro CA, Julia C, Touvier M (2020) Ultraprocessed food consumption and risk of type 2 diabetes among participants of the NutriNet-Santé Prospective Cohort. JAMA Intern Med 180(2):283-291. https://doi.org/10.1001/jamainternmed.2019. 5942

10. Srour B, Fezeu LK, Kesse-Guyot E, Allès B, Méjean C, Andrianasolo RM, Chazelas E, Deschasaux M, Hercberg S, Galan P, Monteiro CA, Julia C, Touvier M (2019) Ultra-processed food intake and risk of cardiovascular disease: prospective cohort study (NutriNet-Santé). BMJ 365:11451. https://doi.org/10.1136/bmj. 11451

11. Schnabel L, Kesse-Guyot E, Allès B, Touvier M, Srour B, Hercberg S, Buscail C, Julia C (2019) Association between ultraprocessed food consumption and risk of mortality among middleaged adults in France. JAMA Intern Med 179(4):490-498. https:// doi.org/10.1001/jamainternmed.2018.7289

12. Vandevijvere S, Jaacks LM, Monteiro CA, Moubarac JC, GirlingButcher M, Lee AC, Pan A, Bentham J, Swinburn B (2019) 
Global trends in ultraprocessed food and drink product sales and their association with adult body mass index trajectories. Obes Rev 20(Suppl 2):10-19. https://doi.org/10.1111/obr.12860

13. Monteiro CA, Moubarac J-C, Levy RB, Canella DS, Louzada MLdC, Cannon G (2018) Household availability of ultra-processed foods and obesity in nineteen European countries. Public Health Nutr 21(1):18-26. https://doi.org/10.1017/S136898001 7001379

14. Vandevijvere S, De Ridder K, Fiolet T, Bel S, Tafforeau J (2019) Consumption of ultra-processed food products and diet quality among children, adolescents and adults in Belgium. Eur J Nutr 58(8):3267-3278. https://doi.org/10.1007/s00394-018-1870-3

15. Calixto Andrade G, Julia C, Deschamps V, Srour B, Hercberg S, Kesse-Guyot E, Allès B, Chazelas E, Deschasaux M, Touvier M, Augusto Monteiro C, Bertazzi Levy R (2021) Consumption of ultra-processed food and its association with sociodemographic characteristics and diet quality in a representative sample of French adults. Nutrients 13(2):682. https://doi.org/10.3390/nu130 20682

16. Costa de Miranda R, Rauber F, de Moraes MM, Afonso C, Santos C, Rodrigues S, Levy RB (2021) Consumption of ultra-processed foods and non-communicable disease-related nutrient profile in Portuguese adults and elderly (2015-2016): the UPPER project. Br J Nutr 125(10):1177-1187. https://doi.org/10.1017/s0007 $11452000344 \mathrm{x}$

17. Adams J, White M (2015) Characterisation of UK diets according to degree of food processing and associations with socio-demographics and obesity: cross-sectional analysis of UK National Diet and Nutrition Survey (2008-12). Int J Behav Nutr Phys Act 12(1):160. https://doi.org/10.1186/s12966-015-0317-y

18. Rauber F, da Costa Louzada ML, Steele EM, Millett C, Monteiro CA, Levy RB (2018) Ultra-processed food consumption and chronic non-communicable diseases-related dietary nutrient profile in the UK (2008-2014). Nutrients 10(5):587. https://doi. org/10.3390/nu10050587

19. European Food Safety Authority (2011) Use of the EFSA comprehensive European food consumption database in exposure assessment. EFSA J 9(3):2097. https://doi.org/10.2903/j.efsa.2011.2097

20. European Food Safety Authority (2015) The food classification and description system FoodEx 2 (revision 2). EFSA Support Publ 12(5):804E. https://doi.org/10.2903/sp.efsa.2015.EN-804

21. Monteiro CA, Levy RB, Claro RM, Castro IR, Cannon G (2010) A new classification of foods based on the extent and purpose of their processing. Cad Saude Publica 26(11):2039-2049. https:// doi.org/10.1590/s0102-311 x2010001100005

22. RIVM (2019) NEVO online version 2019/6.0. https://nevo-online. rivm.nl/

23. van Rossum CTM, Buurma-Rethans EJM, Vennemann FBC, M. B, Brants HAM, de Boer EJ, Ocke MC (2016) The diet of the Dutch : results of the first two years of the Dutch National Food Consumption Survey 2012-2016. Wat eet en drinkt Nederland? : Resultaten van de eerste twee jaren van de Nederlandse voedselconsumptiepeiling 2012-2016. Rijksinstituut voor Volksgezondheid en Milieu RIVM

24. Du H, van der A DL, Boshuizen HC, Forouhi NG, Wareham NJ, Halkjær J, Tjønneland A, Overvad K, Jakobsen MU, Boeing H, Buijsse B, Masala G, Palli D, Sørensen TI, Saris WH, Feskens EJ (2009) Dietary fiber and subsequent changes in body weight and waist circumference in European men and women. Am J Clin Nutr 91(2):329-336. https://doi.org/10.3945/ajen.2009.28191

25. Te Morenga L, Mallard S, Mann J (2013) Dietary sugars and body weight: systematic review and meta-analyses of randomised controlled trials and cohort studies. BMJ 346:e7492. https://doi.org/ 10.1136/bmj.e7492

26. GBD (2019) Risk Factor Collaborators (2020) Global burden of 87 risk factors in 204 countries and territories, 1990-2019: a systematic analysis for the Global Burden of Disease Study 2019. Lancet 396(10258):1223-1249. https://doi.org/10.1016/S01406736(20)30752-2

27. Institute for Health Metrics and Evaluation (2019) GBD Results Tool. University of Washtington, Seattle, USA

28. Pan A, Hu FB (2011) Effects of carbohydrates on satiety: differences between liquid and solid food. Curr Opin Clin Nutr Metab Care 14(4):385-390. https://doi.org/10.1097/MCO.0b013e3283 $46 \mathrm{df} 36$

29. Julia C, Martinez L, Allès B, Touvier M, Hercberg S, Méjean C, Kesse-Guyot E (2018) Contribution of ultra-processed foods in the diet of adults from the French NutriNet-Santé study. Public Health Nutr 21(1):27-37. https://doi.org/10.1017/S136898001 7001367

30. Slimani N, Deharveng G, Southgate DA, Biessy C, Chajès V, van Bakel MM, Boutron-Ruault MC, McTaggart A, Grioni S, Verkaik-Kloosterman J, Huybrechts I, Amiano P, Jenab M, Vignat J, Bouckaert K, Casagrande C, Ferrari P, Zourna P, Trichopoulou A, Wirfält E, Johansson G, Rohrmann S, Illner AK, Barricarte A, Rodríguez L, Touvier M, Niravong M, Mulligan A, Crowe F, Ocké MC, van der Schouw YT, Bendinelli B, Lauria C, Brustad M, Hjartåker A, Tjønneland A, Jensen AM, Riboli E, Bingham S (2009) Contribution of highly industrially processed foods to the nutrient intakes and patterns of middle-aged populations in the European Prospective Investigation into Cancer and Nutrition study. Eur J Clin Nutr 63(Suppl 4):S206-225. https://doi.org/10. 1038/ejen.2009.82

31. Gibney MJ, Forde CG, Mullally D, Gibney ER (2017) Ultra-processed foods in human health: a critical appraisal. Am J Clin Nutr 106(3):717-724. https://doi.org/10.3945/ajen.117.160440

32. Gibney MJ (2018) Ultra-processed foods: definitions and policy issues. Curr Dev Nutr. https://doi.org/10.1093/cdn/nzy077

33. Martínez Steele E, Baraldi LG, Louzada MLdC, Moubarac J-C, Mozaffarian D, Monteiro CA (2016) Ultra-processed foods and added sugars in the US diet: evidence from a nationally representative cross-sectional study. BMJ Open 6(3):e009892. https:// doi.org/10.1136/bmjopen-2015-009892

34. Louzada ML, Martins AP, Canella DS, Baraldi LG, Levy RB, Claro RM, Moubarac JC, Cannon G, Monteiro CA (2015) Impact of ultra-processed foods on micronutrient content in the Brazilian diet. Rev Saude Publica 49:45. https://doi.org/10.1590/s00348910.2015049006211

35. Moubarac J-C, Martins APB, Claro RM, Levy RB, Cannon G, Monteiro CA (2013) Consumption of ultra-processed foods and likely impact on human health. Evidence from Canada. Public Health Nutr 16(12):2240-2248. https://doi.org/10.1017/S1368 980012005009

36. Weaver CM, Dwyer J, Fulgoni VL III, King JC, Leveille GA, MacDonald RS, Ordovas J, Schnakenberg D (2014) Processed foods: contributions to nutrition. Am J Clin Nutr 99(6):15251542. https://doi.org/10.3945/ajen.114.089284

37. Eicher-Miller HA, Fulgoni VL 3rd, Keast DR (2012) Contributions of processed foods to dietary intake in the US from 2003 2008: a report of the Food and Nutrition Science Solutions Joint Task Force of the Academy of Nutrition and Dietetics, American Society for Nutrition, Institute of Food Technologists, and International Food Information Council. J Nutr 142(11):2065S-2072S. https://doi.org/10.3945/jn.112.164442

38. Wang L, Martínez Steele E, Du M, Pomeranz JL, O'Connor LE, Herrick KA, Luo H, Zhang X, Mozaffarian D, Zhang FF (2021) Trends in consumption of ultraprocessed foods among US youths aged 2-19 years, 1999-2018. JAMA 326(6):519-530. https://doi. org/10.1001/jama.2021.10238

39. Baraldi LG, Martinez Steele E, Canella DS, Monteiro CA (2018) Consumption of ultra-processed foods and associated sociodemographic factors in the USA between 2007 and 2012: evidence 
from a nationally representative cross-sectional study. BMJ Open 8(3):e020574. https://doi.org/10.1136/bmjopen-2017-020574

40. Wang L, Steele E, Du M, Mozaffarian D, Zhang FF (2020) Trends in consumption of ultra-processed foods among US children aged 2-19 years, 2003-2016. Curr Dev Nutr 4(Supplement-2):1503-1503. https://doi.org/10.1093/cdn/nzaa061_131

41. FAO (2018) Dietary Assessment: a resource guide to method selection and application in low resource settings. FAO, Rome, Italy
42. European Food Safety Authority (2014) Guidance on the EU Menu methodology. EFSA J 12(12):3944. https://doi.org/10. 2903/j.efsa.2014.3944

43. Willett W (2012) Nutritional epidemiology, vol 40. Oxford University Press, USA 